\title{
Sum Throughput Maximization in a Cognitive Multiple Access Channel With Cooperative Spectrum Sensing and Energy Harvesting
}

\author{
Sinchan Biswas ${ }^{\circledR}$, Student Member, IEEE, Subhrakanti Dey, Senior Member, IEEE, and Amirpasha Shirazinia
}

\begin{abstract}
This paper focuses on the problem of sensing throughput optimization in a fading multiple access cognitive radio (CR) network, where the secondary user (SU) transmitters participate in cooperative spectrum sensing and are capable of harvesting energy and sharing energy with each other. We formulate the optimization problem as a maximization of the expected achievable sum-rate over a finite horizon, subject to an average interference constraint at the primary receiver, peak power constraints, and energy causality constraints at the SU transmitters. The optimization problem is a non-convex, mixed integer non-linear program (MINLP) involving the binary action to sense the spectrum or not, and the continuous variables, such as the transmission power, shared energy, and sensing time. The problem is analyzed under two different assumptions on the available information pattern: 1) non-causal channel state information (CSI), energy state information (ESI), and infinite battery capacity and 2) the more realistic scenario of the causal CSI/ESI and finite battery. In the non-casual case, this problem can be solved by an exhaustive search over the decision variable or an MINLP solver for smaller problem dimensions, and a novel heuristic policy for larger problems, combined with an iterative alternative optimization method for the continuous variables. The causal case with finite battery is optimally solved using a dynamic programming (DP) methodology, whereas a number of sub-optimal algorithms are proposed to reduce the computational complexity of DP. Extensive numerical simulations are carried out to illustrate the performance of the proposed algorithms. One of the main findings indicates that the energy sharing is more beneficial when there is a significant asymmetry between average harvested energy levels/channel gains of different SUs.
\end{abstract}

Index Terms-Energy harvesting, cognitive radio, multiple access channel, spectrum sensing, fading channel.

\section{INTRODUCTION}

$\mathbf{T}$ WO OF the essential and limited resources in wireless communications are radio spectrum and energy. With the advent of advanced data hungry mobile devices, the demands for both of them have increased significantly. The demands

Manuscript received September 30, 2018; revised February 6, 2019; accepted March 23, 2019. Date of publication April 2, 2019; date of current version June 7, 2019. This work was supported by Swedish Research Council Project grant no: 2017-04053. A related work [39] has been published in EUSIPCO 2016. The associate editor coordinating the review of this paper and approving it for publication was R. Schober. (Corresponding author: Sinchan Biswas.)

S. Biswas and S. Dey are with the Division of Signals and Systems, Uppsala University, 75105 Uppsala, Sweden (e-mail: sinchan.biswas@ angstrom.uu.se).

A. Shirazinia is with Analytics and AI Group, Swedbank, 17062 Stockholm, Sweden.

Digital Object Identifier 10.1109/TCCN.2019.2908860 for spectrum usage have been increasing due to applications consuming high bandwidth. The traditional rigid spectrum allocation policy is found to be fairly inefficient to mitigate this problem [1]. To combat this issue, a dynamic spectrum allocation policy paradigm of cognitive radio (CR) network has been proposed in the literature [2]. In particular, in the interweave paradigm of $\mathrm{CR}$, the unlicensed secondary users (SU) are capable of actively sensing the licensed primary user (PU) spectrum and using it whenever it is detected to be not utilized by the PU [3]. Similarly, the SUs have to vacate the PU spectrum as soon as it senses PU activity. In such a framework, sensing of PU spectrum has become a significant task. Significant research has been focused on different spectrum sensing algorithms like energy detection, cyclostationary detection, matched filter detection etc [4]. The main focus of such algorithms is to increase the efficiency of spectrum utilization, while protecting PU from harmful interference. In a multiuser secondary network with varying degree of sensing performances, it has been well established that cooperative spectrum sensing improves the PU detection performance. A contemporary survey of energy-efficient cooperative spectrum sensing algorithms can be found in [5] (see also references therein).

Alongside spectrum utilization efficiency, the efficiency in utilizing the energy has also become a significant issue. Just like the spectrum requirement, the communication overhead, signal transmission and detection at the receiver all require significant amounts of energy depending on the application. The usual battery powered electrical sources are unable to fully replenish these ever-growing requirements because of its non-renewable feature. In many applications, it is difficult to replace the batteries attached to the wireless devices periodically because of their remote locations and the required labour costs. To address this issue, the usage of renewable energy has been proposed in the literature [6]. Such energy from the wind, solar or other unconventional power sources are not only efficient in terms of fulfilling the energy requirement but environmentally sustainable as well. This has led to extensive research activities in the domain of energy harvesting wireless communications and networking [7], [8].

Significant recent research has been directed towards the utilization of energy harvesting in wireless networks. The transmission policies have been investigated for minimization of transmission time completion with infinite battery capacity [9] and finite sized battery [10]. Similar problems have 
been addressed in the context of different channel models. The optimal policies have been derived in the context of multiuser settings with relay channel [11], broadcast channels [12], interference channels [13] and multiple access channels [14].

CR network with energy harvesting capabilities effectively use both of the above mentioned resources, which is the focus of the present work. There has been significant research in this domain. Opportunistic channel access and energy harvesting process has been studied in this framework in [15], [16]. Optimal spectrum sensing policy in an energy harvesting CR network has been investigated in [17]. Opportunities and challenges in such a network has been surveyed in [18]. The energy harvesting feature creates a reliable energy efficient system, while the dynamic spectrum allocation leads to more efficient spectrum utilization. The cooperative energy sharing feature has been incorporated into the system to satisfy the energy requirements of the more vulnerable users in terms of average harvested energy.

\section{A. Background}

This paper focuses on the specific case of CR networks with SUs having energy harvesting and sharing capabilities. The channels between SUs and the fusion centre (FC) constitute a fading multiple access channel. For such a network, the achievable sum-rate is generally used as a throughput measure. Optimizing the achievable sum-rate in a CR network has been investigated with respect to different fading channel models in [19], [20]. As spectrum sensing is one of the primary functionalities in CR networks, the sensing throughput tradeoff has been rigorously studied in [21]. Capacity analysis of wireless systems with energy harvesting capabilities has been studied as well in [22]. Such analysis has also been extended to the CR networks in the literature [23], [24]. In a separate field of literature, distortion minimization in a multi-sensor setting has been studied in an energy harvesting wireless sensor network (WSN) with wireless energy transfer mechanisms [25].

There has been some recent research in CR networks with SUs having energy harvesting capabilities. In [26], the spectrum sensing strategy is studied for a system with nonlinear energy harvesting model. In [27], achievable throughput optimization has been investigated with respect to sensing time and sensing threshold, whereas [28] addresses the problem of optimizing the throughput with respect to sensing energy, transmission energy and spectrum sensing time. In [29], the authors analyze the asymptotic activity behavior of a single SU in a hybrid energy harvesting scenario, and subsequently analyze the optimal sum throughput with respect to optimization parameters such as asymptotic active probabilities, sensing durations and detection thresholds over a single time slot for a collection of heterogenous SUs, followed by an algorithm for selecting the best subset of cooperating SUs. In [30], the authors of [29] tackled the problem of throughput optimization with different sets of fusion rules. In [31], the same authors addressed the issue of protecting PU from collision with SU traffic. In [32], the cross-layer problem involving physical layer transmission power and network layer delay is addressed with a game-theoretic formulation. In [33], throughput is optimized with respect to the sensing threshold and the amount of energy being harvested. In [34], the problem of optimal spectrum sensing strategy is studied in energy harvesting CR networks. In [35], this optimization is investigated with respect to sensing time and energy harvesting time, considering a slotted energy arrival policy. In [36], the problem is studied in the scenario of multislot spectrum sensing and energy harvesting. In [37], the optimal energy scheduling problem is studied in the fading multiple access channel with energy harvesting. In [38], optimal power allocation policy is analyzed when only causal information is available to the energy harvesting transmitters.

We partially addressed the problem of throughput optimization in a CR fading multiple access channel with energy harvesting secondary transmitters in [39] in a cooperative spectrum sensing framework. It is obvious that there is a trade-off between the accuracy of spectrum sensing process and the throughput measure. This is due to the fact that, if the sensing time is long enough, then the sensing accuracy is high, but on the other hand, the transmission time decreases, which in turn reduces the throughput. In [39], the optimization was carried out with respect to the sensing time, SU transmission power, and the binary decision variables concerning (i) whether to sense (as sensing also costs energy) and (ii) the outcome of the spectrum sensing decision regarding whether to access the spectrum or not. In our current submission, we remove the binary variable regarding the spectrum access decision by considering explicit expressions for detection and false alarm probability for the cooperative spectrum sensing process, and we extend the paper to consider the additional feature of energy sharing between neighbouring sensors. The energy sharing mechanism considered here is assumed to be directional, which can be achieved via non radiofrequency wireless power transfer over shorter distances or radio frequency based wireless power transfer over longer distances employing energy beamforming technology [40], [41]. In addition, as opposed to [39], where the PU interference at the secondary base station was ignored, we consider the presence of PU interference explicitly in the SU sum-throughput expression when the PU is present. In this setting, we study the problem of jointly maximizing the achievable sum-rate for a CR fading multiple access channel with respect to the binary decision variables regarding whether each SU senses for the $P U$, and the continuous variables such as the $S U$ transmission powers, sensing times and energy transferred between neighbouring sensors under an average interference constraint at the PU receiver, energy causality and peak power constraints at the SU transmitters.

\section{B. Contributions}

We consider the problem of maximizing the achievable sumrate with respect to the four optimization variables mentioned above. The specific contributions of this paper are listed below:

- We solve the problem of expected sum throughput maximization over a finite time horizon in a fading multiple access cognitive network where the SUs having energy harvesting and sharing capabilities. We explore 
the problem in both finite and infinite battery capacity settings, and with non-causal and causal information patterns. In the case where all channel conditions and harvested energy realizations are known non-causally for the entire time horizon, an analysis is provided for the optimization of the continuous variables (SU transmission power, shared energy and sensing times) for the infinite battery capacity setting, using an iterative alternating optimization approach, which only ensures locally optimal solutions, as the problem is jointly non-convex in the continuous variables.

- In case of a non-causal information pattern, the overall optimization problem is a mixed integer nonlinear programming problem, where the underlying nonlinear programming problem is non-convex. It is well known that (see [42], [43]) determining of a global solution of the non-convex MINLP problem is NP-hard. We do not pursue global optimization methods to avoid their prohibitive computational complexity in the case of longer horizons. Instead, for smaller problem dimensions (small number of SUs and a short time horizon), we use an exhaustive search policy to find the optimal binary variables regarding the SUs' decision to sense the spectrum. We also provide a numerical study comparing the results with those obtained using a global optimization software. For larger problem dimensions, using either the exhaustive search or global optimization is impractical, and therefore we propose a heuristic policy to solve the mixed integer non-linear program with respect to the spectrum sensing decisions resulting in a moderate computational complexity.

- The problem pertaining to the finite battery capacity with causal CSI and ESI is studied using a dynamic programming based stochastic control algorithm resulting in the optimal sequence of the binary spectrum sensing decision variables, and discretized values of the continuous variables in order to facilitate practical implementation.

- We also propose two other sub-optimal policies to mitigate the exponential complexity associated with the dynamic programming (DP) algorithm. The first one is a moving-horizon limited look-ahead policy, which has a relatively low complexity especially when the length of the finite horizon is high. The second one is an ad-hoc policy to find sub-optimal values of all three continuous optimization variables without involving the dynamic programming algorithm, based on insights obtained from the non-causal case.

- Finally, we compare a non-adaptive policy for sensing time optimization (i.e., a fixed sensing time is adopted across all time slots) with an adaptive sensing time optimization (where the sensing time is optimized in a dynamical fashion across the time slots) in the above mentioned setting.

\section{Organization}

The rest of the paper is organized as follows. In Section II we discuss the system model. In Section III we describe the

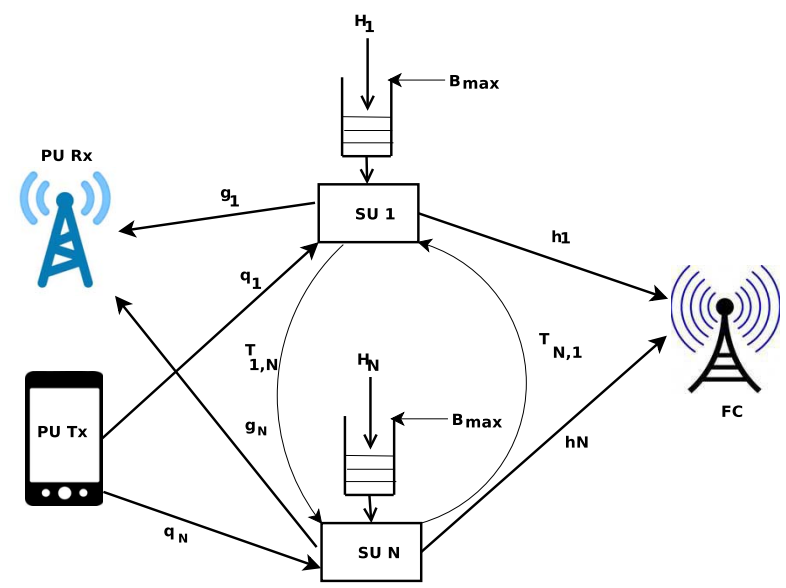

Fig. 1. Figure of System Model.

optimization problem and the procedure for finding the optimal solution. In Section IV we provide an analysis for the optimal solution of the problem for the infinite battery and noncausal CSI/ESI scenario. In Section V we discuss the proposed policy for causal CSI and finite battery scenario using the dynamic programming algorithm. In Section VI we propose two sub-optimal policies in order to mitigate the complexity of dynamic programming algorithm. Section VII contains simulation results followed by some concluding remarks in Section VIII.

\section{SySTEM MODEL}

We consider a system model (see Figure 1) comprised of a PU, $N$ SUs and a FC, the role of which can be performed by a secondary base station (SBS). We adopt a cooperative spectrum sensing model, where individual SUs sense the PU spectrum locally and transmit their local decisions to the SBS. The SBS collates all the information from the SUs and arrives at a global decision, which in turn is broadcast to the SUs by a control channel, so that the SUs can decide to transmit or not. We assume that time is slotted as shown in Fig. 2. the energy harvesting and sharing process is assumed to be independent of the sensing and data transmission process, as we do not restrict the harvesting and sharing to be based on RF energy harvesting only, and these actions are carried out by the sensor via a unit separate from the transceiver. We consider a finite time horizon of $M$ slots, where each slot is assumed to be $T$ time units in size. In each slot, the PU is either present with probability $\mu$ or absent with probability $(1-\mu)$. In the $k^{t h}$ time slot, as illustrated in Fig. 2, $\tau_{k}$ time units are used for the spectrum sensing process and the residual $\left(T-\tau_{k}\right)$ time units are used for the transmission process, provided the PU spectrum is found to be vacant. Since sensing costs energy, in the $k^{t h}$ time slot, the $i^{\text {th }} \mathrm{SU}$ decides to either sense the PU spectrum or be idle based on the binary decision variable $a_{i, k}, 1 \leq i \leq N, 1 \leq k \leq M . a_{i, k} \in\{0,1\}$, where 1 (or 0 ) represents the decision to sense the PU spectrum (or not). Note that if $B_{i, k}$, the battery energy level of the $i^{\text {th }} \mathrm{SU}$ at the beginning of the $k^{\text {th }}$ time slot, is smaller than $p_{s} \tau_{k}$, where 


\begin{tabular}{|l|l|}
\hline Sensing time $\tau_{k}$ & Transmission time $\left(T-\tau_{k}\right)$ \\
\hline
\end{tabular}

Time Slot: $T$ time units

Energy harvesting and sharing Process

Fig. 2. Frame Structure.

$p_{s}$ is the sensing power, $a_{i, k}=0$. However, if $B_{i, k}>p_{s} \tau_{k}$, $a_{i, k} \in\{0,1\}$.

\section{A. Energy Harvesting and Battery Dynamics}

As indicated in Fig. 2, we assume an energy full-duplex energy harvesting scenario [29], where each SU has separate energy harvesting/sharing units and spectrum sensing and information transmitter/receivers so that these harvesting/energy sharing and spectrum sensing/information transmission/reception processes can occur simultaneously and independently. This is not unusual where the SU can harvest energy from non-radio frequency sources, such as solar, wind or vibrations etc. We also make the standard assumption that the energy harvested in slot $k$ is available for use in slot $k+1$ onward.

When the spectrum sensing decision $a_{i, k}=1$, the SU transmits with a transmission power $p_{i, k}$. With the assumption of a finite battery size of $B_{\max }$ at each SU, $B_{i, k}$ can be expressed as follows:

$$
\begin{aligned}
B_{i, k+1}=\min & \left\{B_{\max }, B_{i, k}-E_{i, k}+H_{i, k}-\sum_{m \in \mathcal{N}_{T, i}} T_{i, m}(k)\right. \\
& \left.+\sum_{m \in \mathcal{N}_{R, i}} \eta_{m, i} T_{m, i}(k)\right\}
\end{aligned}
$$

where $H_{i, k}$ is a random process denoting the amount of energy harvested by the $i^{\text {th }} \mathrm{SU}$ in the $k^{\text {th }}$ time slot. $T_{i, m}(k)$ is the amount of energy transferred from the $i^{\text {th }} \mathrm{SU}$ to the $m^{\text {th }}$ SU in the $k^{t h}$ time slot. $E_{i, k}, \eta_{m, i}, \mathcal{N}_{T, i}, \mathcal{N}_{R, i}$ represents the amount of energy used by the $i^{\text {th }} \mathrm{SU}$ in the $k^{\text {th }}$ time slot, the energy transfer efficiency from the $m^{\text {th }} \mathrm{SU}$ to the $i^{\text {th }} \mathrm{SU}$, the set of SUs which $i^{\text {th }}$ SU shares its energy to and the set of SUs that the $i^{\text {th }} \mathrm{SU}$ received shared energy from, respectively. Although our subsequent analysis does not strictly need it, we assume that $H_{i, k}$ is independent and identically distributed (i.i.d.) across time and independent across the $\mathrm{SU}$ terminals.

In the case where $a_{i, k}$ is a variable of optimization, $E_{i, k}$, can be subdivided into sensing energy and transmission energy as follows:

$$
\begin{aligned}
E_{i, k}= & \left\{a_{i, k} p_{s} \tau_{k}+p_{i, k}\left(T-\tau_{k}\right)\right. \\
& \left.\times\left\{\left(1-P_{d}\left(\tau_{k}\right)\right) \mu+\left(1-P_{f a}\left(\tau_{k}\right)\right)(1-\mu)\right\}\right\}
\end{aligned}
$$

where $P_{d}\left(\tau_{k}\right)$ and $P_{f a}\left(\tau_{k}\right)$ denote the probability of detection and the probability of false alarm at the FC in the $k^{\text {th }}$ time slot, respectively. Note that in order to avoid an additional binary decision variable regarding whether to access the spectrum or not, we consider the above energy consumption model, which is equivalent to accessing the spectrum with probability $\left(1-P_{d}\left(\tau_{k}\right)\right) \mu+\left(1-P_{f a}\left(\tau_{k}\right)\right)(1-\mu)$ in the $k^{\text {th }}$ slot. This approximation becomes exact when the time horizon $M$ becomes sufficiently large. See [39] for a treatment where the spectrum access decision is also treated as a binary variable.

Applying the recursive definition of (1) we can write:

$$
\begin{aligned}
B_{i, k}=\min & \left\{B_{m a x}, B_{i}-\sum_{r=1}^{k} E_{i, r}+\sum_{r=1}^{k-1} H_{i, r}-\sum_{r=1}^{k} \sum_{m \in \mathcal{N}_{T, i}}\right. \\
& \left.\times T_{i, m}(r)+\sum_{r=1}^{k-1} \sum_{m \in \mathcal{N}_{R, i}} \eta_{m, i} T_{m, i}(r)\right\}
\end{aligned}
$$

where $E_{i, k}$ is defined in (2) and $B_{i}$ represents the initial battery state for the $i^{\text {th }} \mathrm{SU}$. In the case of infinite battery capacity, the above expression in (3) simplifies to:

$$
\begin{aligned}
B_{i, k}= & B_{i}-\sum_{r=1}^{k} E_{i, r}+\sum_{r=1}^{k-1} H_{i, r}-\sum_{r=1}^{k} \sum_{m \in \mathcal{N}_{T, i}} T_{i, m}(r) \\
& +\sum_{r=1}^{k-1} \sum_{m \in \mathcal{N}_{R, i}} \eta_{m, i} T_{m, i}(r)
\end{aligned}
$$

\section{B. Spectrum Sensing Model}

If an SU decides to sense the PU spectrum, the spectrum sensing time $\tau_{k}$ for the $k^{\text {th }}$ time slot is divided into a number of mini-slots, where the length of each mini-slot is pre-decided. In each mini-slot the SUs take sensing samples and try to determine the PU spectrum availability by using a binary hypothesis testing problem based on the following signal model:

$$
\begin{aligned}
& \mathcal{H}_{0}: y_{i, k, m}=n_{i, k, m} \\
& \mathcal{H}_{1}: y_{i, k, m}=\sqrt{q_{i}} x_{k}+n_{i, k, m}
\end{aligned}
$$

where $x_{k}$ is the PU transmitted signal for the $k^{\text {th }}$ time slot, which is real valued and distributed as $x_{k} \sim \mathcal{N}\left(0, \sigma_{x}^{2}\right)$. $q_{i}$ is the channel power gain between the PU transmitter and the $i^{\text {th }}$ $\mathrm{SU}$ receiver, which is assumed to be constant throughout the sensing process. $y_{i, k, m}$ and $n_{i, k, m}$ are the real valued received signal and noise signal component respectively at the $i^{\text {th }} \mathrm{SU}$ receiver for the $m^{t h}$ mini-slot of the $k^{t h}$ time slot. The noise is distributed i.i.d. as $n_{i, k, m} \sim \mathcal{N}\left(0, \sigma_{n}^{2}\right)$. For the spectrum sensing rule, we use an energy detection policy [44] in each $\mathrm{SU}$, such that the local spectrum sensing decision at the $k^{t h}$ time slot in the $i^{\text {th }} \mathrm{SU}$ is determined by the following rule:

$$
\theta_{i, k}=\mathcal{I}\left(\frac{1}{\mathcal{S}_{k}} \sum_{m=1}^{\mathcal{S}_{k}} y_{i, k, m}^{2} \geq \epsilon\right)
$$

where $\mathcal{I}$ is the indicator function, $\mathcal{S}_{k}$ is the number of minislots in a particular time slot $\tau_{k}$ and $\epsilon$ is the detection threshold. However, we should note the fact that the number 
of mini-slots $\mathcal{S}_{k}$ being a discrete variable doesn't imply that the spectrum sensing time $\tau_{k}$ being a discrete variable. Thus in general if the length of each mini-slot is $\tau_{m i n i}$, a continuous variable, the corresponding number of mini-slots can be determined as $\mathcal{S}_{k}=\left\lfloor\frac{\tau_{k}}{\tau_{m i n i}}\right\rfloor$. All the local decisions $\theta_{i, k}$ from the SUs are sent to the FC by control channels and collated using an OR logic fusion rule to achieve the global decision $\theta_{k}$. Note once again that our analysis is not restrictive to any particular type of a fusion rule and other fusion rules such as the "majority logic" rule can also be easily considered (see Remark 2 below).

Remark 1: A number of additional sources of energy consumption has been ignored in this work, such as circuit power consumption, energy consumption due to signalling overhead of side-information over control channels etc. The rationale is that (i) the circuit power consumption is assumed to be much lower compared to energy consumption in communication, especially over longer distances, and (ii) a non-trivial circuit power consumption can be grouped together with the sensing energy (whenever $a_{i, k}=1$ ) whereas energy consumption due to signalling overhead can be easily subtracted from the maximum battery capacity without affecting the analysis. The nature of the numerical results will not be affected considerably as long as such energy consumption is small compared to energy consumed due to communication.

The probability of detection and false alarm for the OR fusion rule then can be written as $P_{d}=\operatorname{Pr}\left\{\theta_{k}=1 \mid \mathcal{H}_{1}\right\}$ and $P_{f a}=\operatorname{Pr}\left\{\theta_{k}=1 \mid \mathcal{H}_{0}\right\} . P_{d}\left(\tau_{k}\right)$ and $P_{f a}\left(\tau_{k}\right)$ is given by the following:

$$
\begin{gathered}
P_{d}\left(\tau_{k}\right)=1-\Pi_{i \in \mathcal{A}_{k}}\left(1-p_{d}^{(i)}\left(\tau_{k}\right)\right) \\
P_{f a}\left(\tau_{k}\right)=1-\Pi_{i \in \mathcal{A}_{k}}\left(1-p_{f a}^{(i)}\left(\tau_{k}\right)\right)
\end{gathered}
$$

where $\mathcal{A}_{k}$ is defined as the set $\mathcal{A}_{k}=\left\{i: a_{i, k}=1\right\}$, and $p_{d}^{(i)}\left(\tau_{k}\right)$ and $p_{f a}^{(i)}\left(\tau_{k}\right)$ are the probability of detection and the probability of false alarm at the $i^{\text {th }} \mathrm{SU}$ in $k^{\text {th }}$ time slot, given the sensing time $\tau_{k}$ and they can be computed by the following expressions [21]:

$$
\begin{aligned}
& p_{d}^{(i)}\left(\tau_{k}\right)=\mathbf{Q}\left\{\left(\frac{\epsilon}{\sigma_{n}^{2}}-\gamma_{i}-1\right) \sqrt{\frac{\tau_{k} f_{s}}{2 \gamma_{i}+1}}\right\} \\
& p_{f a}^{(i)}\left(\tau_{k}\right)=\mathbf{Q}\left\{\left(\frac{\epsilon}{\sigma_{n}^{2}}-1\right) \sqrt{\tau_{k} f_{s}}\right\}
\end{aligned}
$$

In the above two equations $f_{s}$ is the sampling rate and $\gamma_{i}=$ $\frac{q_{i}}{\sigma_{n}^{2}}$ is the signal to noise ratio corresponding to the channel between the PU transmitter and the $i^{\text {th }} \mathrm{SU}$ receiver.

Remark 2: If majority logic fusion rule is implemented with identical detection and false alarm probabilities $p_{d}, p_{f a}$ respectively, for all SUs, then $P_{d}$ and $P_{f a}$ can be calculated as follows:

\begin{tabular}{|c|c|}
\hline$\tau_{k}$ & $\begin{array}{l}\text { Time taken to perform the spectrum sensing in the } k^{t h} \\
\text { slot }\end{array}$ \\
\hline$p_{i, k}$ & Transmission power for the $i^{t h} \mathrm{SU}$ in the $k^{t h}$ time slot \\
\hline$T_{i, m}(k)$ & $\begin{array}{l}\text { Energy shared from the } i^{t h} \mathrm{SU} \text { to the } m^{t h} \mathrm{SU} \text { in the } \\
k^{t h} \text { time slot }\end{array}$ \\
\hline$\mu$ & PU activity probability \\
\hline $\mathcal{N}_{T, i}$ & Set of SUs that the $i^{t h}$ SU shares its energy to \\
\hline $\mathcal{N}_{R, i}$ & Set of SUs that the $i^{t h}$ SU receives shared energy from \\
\hline$\eta_{m, i}$ & $\begin{array}{l}\text { Energy transfer efficiency from the } m^{t h} \mathrm{SU} \text { to the } i^{\text {th }} \\
\text { SU }\end{array}$ \\
\hline$h_{i, k}$ & $\begin{array}{l}\text { Channel gain between the } i^{t h} \text { SU transmitter and the } \\
\text { FC in the } k^{t h} \text { time slot }\end{array}$ \\
\hline$g_{i, k}$ & $\begin{array}{l}\text { Channel gain between the } i^{t h} \mathrm{SU} \text { transmitter and the } \\
\text { PU Receiver in the } k^{\text {th }} \text { time slot }\end{array}$ \\
\hline$q_{i}$ & $\begin{array}{l}\text { Channel power gain between the PU transmitter and } \\
\text { and the } i^{t h} \mathrm{SU}\end{array}$ \\
\hline$p_{s}$ & Power required to sense for the single unit of time \\
\hline$P_{\max }$ & Peak power limit on $p_{i, k}$ \\
\hline$B_{i}$ & Initial battery state for the $i^{t h} \mathrm{SU}$ \\
\hline$H_{i, k}$ & Energy harvested for the $i^{t h} \mathrm{SU}$ for the $k^{t h}$ time slot \\
\hline$a_{i, k}$ & $\begin{array}{l}\text { Decision to sense the spectrum or not for the } i^{t h} \mathrm{SU} \\
\text { in } k^{t h} \text { time slot }\end{array}$ \\
\hline$Q_{\text {avg }}$ & Average interference limit to the PU \\
\hline$\sigma^{2}$ & $\begin{array}{l}\text { Noise variance in the channel between the SU trans- } \\
\text { mitter and the FC }\end{array}$ \\
\hline$\sigma_{i n}^{2}$ & Interference caused by the PU presence \\
\hline
\end{tabular}

$$
\begin{aligned}
P_{d}\left(\tau_{k}\right) & =\sum_{l=\left\lfloor\left|\mathcal{A}_{k}\right| / 2\right\rfloor+1}^{\left|\mathcal{A}_{k}\right|}\left(\begin{array}{c}
\left|\mathcal{A}_{k}\right| \\
l
\end{array}\right) p_{d}^{l}\left(1-p_{d}\right)^{\left|\mathcal{A}_{k}\right|-l} \\
P_{f a}\left(\tau_{k}\right) & =\sum_{l=\left\lfloor\left|\mathcal{A}_{k}\right| / 2\right\rfloor+1}^{\left|\mathcal{A}_{k}\right|}\left(\begin{array}{c}
\left|\mathcal{A}_{k}\right| \\
l
\end{array}\right) p_{f a}^{l}\left(1-p_{f a}\right)^{\left|\mathcal{A}_{k}\right|-l}
\end{aligned}
$$

TABLE I

SYSTEM PARAMETERS

where $\left|\mathcal{A}_{k}\right|$ denotes the cardinality of the set $\mathcal{A}_{k}$, and $\lfloor x\rfloor$ denotes the nearest integer less than $x$.

\section{Communication Channel Models}

We assume that the spectrum sensing channel gain $\left(q_{i}\right)$, and the communication channel gains between the SU transmitters and PU receiver $\left(g_{i, k}\right)$, as well as the SU transmitters and the FC $\left(h_{i, k}\right)$ are i.i.d. exponentially distributed with unity mean unless otherwise stated. All channel gains are assumed to be mutually statistically independent. Interference caused by the $\mathrm{PU}$ at the $\mathrm{FC}$ is treated as noise.

For easier readability we have summarized the relevant parameters for our subsequent problem formulation in Table I.

\section{Problem Formulation}

In this section, we formulate the optimization problem considered in this paper, namely, an achievable sum rate maximization for a SU multiple-access channel, under the above mentioned cooperative spectrum sensing framework, with an average interference constraint [45] at the PU receiver, and energy harvesting as well as peak transmit power constraints at the SU transmitters.

\section{A. Achievable Sum Rate Maximization}

The achievable sum-rate expression, also weighted by the probability of spectrum access, has two components. One component corresponds to the case when the PU is active but not detected by the spectrum sensing process, and the other one corresponds to when it is not active and is also correctly detected to be idle. The respective weighted achievable 
sum-rate can be expressed as the following:

$$
\begin{aligned}
\mathcal{D}_{1}\left(p_{i, k}, \tau_{k}\right)= & \frac{\mu}{M} \frac{T-\tau_{k}}{T}\left(1-P_{d}\left(\tau_{k}\right)\right) \\
& \times \log _{2}\left\{1+\frac{\sum_{i=1}^{N} p_{i, k} h_{i, k}}{\sigma^{2}+\sigma_{i n}^{2}}\right\} \\
\mathcal{D}_{2}\left(p_{i, k}, \tau_{k}\right)= & \frac{1-\mu}{M} \frac{T-\tau_{k}}{T}\left(1-P_{f a}\left(\tau_{k}\right)\right) \\
& \times \log _{2}\left\{1+\frac{\sum_{i=1}^{N} p_{i, k} h_{i, k}}{\sigma^{2}}\right\}
\end{aligned}
$$

where the PU interference (treated as noise) is given by $\sigma_{i n}^{2}$. The sensing throughput maximization problem can be formulated as the following (for all $1 \leq i \leq N, 1 \leq k \leq M$ ):

$$
\begin{aligned}
& \max _{a_{i, k}, p_{i, k}, \tau_{k}, T_{i, m}(k)} \mathbf{E}\left\{\sum_{k=1}^{M}\left\{\mathcal{D}_{1}\left(p_{i, k}, \tau_{k}\right)+\mathcal{D}_{2}\left(p_{i, k}, \tau_{k}\right)\right\}\right\} \\
& \text { s.t. } \frac{1}{M} \sum_{k=1}^{M} \mathbf{E}\left\{\frac{T-\tau_{k}}{T}\left(1-P_{d}\left(\tau_{k}\right)\right) \sum_{i=1}^{N} p_{i, k} g_{i, k}\right\} \leq Q_{a v g} \\
& 0 \leq p_{i, k} \leq P_{m a x} ; \forall i, k ; 0 \leq \tau_{k} \leq T ; \forall k \\
& 0 \leq T_{i, m}(k) \forall i, m, k \\
& \sum_{r=1}^{k}\left(a_{i, r} p_{s} \tau_{r}+p_{i, r}\left(T-\tau_{r}\right)\left(\left(1-P_{d}\left(\tau_{r}\right)\right) \mu\right.\right. \\
& \left.\left.\quad+\left(1-P_{f a}\left(\tau_{r}\right)\right)(1-\mu)\right)\right) \leq B_{i}+\sum_{r=1}^{k-1} H_{i, r} \\
& -\sum_{r=1}^{k} \sum_{m \in \mathcal{N}_{T, i}} T_{i, m}(r)+\sum_{r=1}^{k-1} \sum_{m \in \mathcal{N}_{R, i}} \eta_{m, i} T_{m, i}(r) \text { a.s.; } \forall i, k \\
& a_{i, k}= \begin{cases}0 & \text { if } B_{i, k}<p_{s} \tau_{k} \\
\in\{0,1\} & \text { otherwise }\end{cases}
\end{aligned}
$$

where a.s. stands for almost surely.

Remark: It is important to note that the average interference term in constraint has been normalized by the primary activity factor $\mu$, where $Q_{a v g}=Q / \mu, Q$ being the true average interference limit, since no interference is caused when the primary is not active. We would also like to point out that the achievable sum-throughput expression used in the above optimization problem for the multiple access channel has the underlying assumption that the codewords used for data transmission at each SU are independently generated from the corresponding statistically independent Gaussian codebooks [46], followed by successive decoding at the receiver.

The problem under consideration is a joint optimization with respect to the transmission power $p_{i, k}$, transferred energy $T_{i, m}(k)$, sensing time $\tau_{k}$ and the decision to sense $a_{i, k}$. The objective is to maximize the weighted achievable sum rate expression (8), with respect to an average interference constraint (9) imposed over the entire time horizon, whereas (10) signifies the short term transmission power and sensing time and transferred energy constraints imposed at each time slot. The last constraint (11) is the energy causality constraint which ensures for every $\mathrm{SU}, E_{i, k} \leq B_{i, k} \quad \forall i, k$, and states that $a_{i, k}=0$ (no sensing is possible) when there is not enough energy to sense in the beginning of the $k$-th time slot, whereas $a_{i, k} \in\{0,1\}$ otherwise.

This optimization problem is a mixed-integer nonlinear programming (MINLP) problem with respect to the binary variable $a_{i, k}$, and the continuous variables $p_{i, k}, \tau_{k}$ and $T_{i, m}(k)$. In this particular case, the nonlinear programming problem with respect to the continuous variables is non-convex, and hence the associated MINLP problem is NP-hard [42], [47]. In the non-causal case where all channel and harvested energy information is known a priori, one can still use global optimization tools, which demand exponential computational complexity with respect to $M, N$. Consequently, for the case of small $M, N$, we use an exhaustive search method to determine $a_{i, k}$, followed by an alternative iterative optimization method for the continuous optimization variables as described below. Noting that for a fixed $\tau_{k}$, the resulting problem becomes jointly convex in $p_{i, k}, T_{i, m}(k)$ and can be solved efficiently. On the other hand for fixed $p_{i, k}$ and $T_{i, m}(k)$, it results in a non-convex problem in $\tau_{k}$, which can also be locally optimized. This iterative process is continued until a locally optimum solution is found and the algorithm converges within a certain tolerance. We compare the performance of this approach with a global optimization tool based solution and reveal that the corresponding solutions are very close. For large values of $M, N$, both approaches quickly fail to be scalable. Note however, that a global optimization approach cannot be used in the causal case, as this involves a dynamic programming based approach, where the associated value functions at each iteration of the dynamic programming do not have a closed form expression.

\section{B. Heuristic Policy for $a_{i, k}$}

In the case of medium to large $M, N$, one cannot employ the exhaustive search method to optimize over the binary variable $a_{i, k}$. Instead, we propose a heuristic policy to determine $a_{i, k}$. The policy focuses on the fact that an upper bound on probability of false alarm, i.e., $P_{f a} \leq \kappa$, for some $\kappa>0$, which, in turn imposes a lower bound on sensing time as $\tau_{l} \leq \tau_{k}$. The value of such a lower bound $\tau_{l}$ can be determined according to [21] $\tau_{l}=\frac{1}{f_{s}}\left\{\frac{\mathbf{Q}^{-1}\left(P_{f a}\right)}{\frac{\epsilon}{\sigma_{f}^{2}}-1}\right\}^{2}$, where $\mathbf{Q}$ is the cumulative distribution function of the standard Gaussian random variable. This heuristic policy proposes that if a particular SU has more than enough energy in its battery to perform the spectrum sensing operation for $\tau_{l}$ time units, the decision to sense is taken affirmatively, i.e., $a_{i, k}=1$, and vice versa. It should be noted that the decision making of spectrum access decision variable $a_{i, k}$ for this sub-optimal heuristic policy is taken by individual SUs without any centralized control, which is different from the optimal policy. Thus for this policy, if a particular SU decides not to sense, i.e., $a_{i, k}=0$, implying that the $i$-th SU doesn't have enough energy for sensing for the $k$-th time slot, therefore setting the transmission power $p_{i, k}=0$. In the optimal policy however, $a_{i, k}=0$ does not necessarily imply $p_{i, k}=0$, as an SU may choose not to participate in sensing, but still may access the spectrum if dictated by the optimum solution. 
In the following section we analyze the optimization problem in the context of non-causal CSI and ESI and infinite battery capacity. For a general $M$-horizon problem we provide an analysis for determining $p_{i, k}$ and $T_{i, m}(k)$ for a fixed $\tau_{k}$, and discuss feasibility conditions for finding a locally optimal sensing time given fixed $p_{i, k}$ and $T_{i, m}(k)$.

\section{Non-Causal Optimization With INFINITE BATTERY}

We discuss the non-causal throughput maximization problem over a finite time horizon in the context of infinite battery in two different settings: adaptive and non-adaptive sensing time scenarios. Section IV-A focuses on the adaptive sensing time strategy, where the sensing time is optimized for each time slot, whereas Section IV-B describes the algorithm for the optimization. We study the non-adaptive sensing time optimization strategy in Section IV-C, where the sensing time is kept at a fixed optimized value for all time slots.

\section{A. Adaptive Optimization With Non-Causal CSI}

In this subsection we derive the optimal transmission power $\left(p_{i, k}\right)$ and shared energy $\left(T_{i, m}(k)\right)$ while keeping the sensing time $\tau_{k}$ fixed. The optimization problem under consideration is then a jointly convex problem in $p_{i, k}$ and $T_{i, m},(k)$. In the following subsection, we derive the optimal sensing time keeping $p_{i, k}$ and $T_{i, m}(k)$ fixed. The channel state information $g_{i, k}, h_{i, k}$ and the energy state information of the battery $H_{i, k}$ are assumed to be known non-causally for this scenario. The following derivation assumes that $a_{i, k}$ is pre-determined either according to an exhaustive search strategy or the heuristic policy proposed in Section III-B. The optimal values are determined using the KKT necessary conditions. As the overall problem is non-convex, only local optima can be guaranteed.

1) Transmission Power and Transferred Energy Optimization: We first provide an analysis for obtaining the optimal transmission power and shared energy parameters in the context of non-causal CSI and ESI, assuming the battery capacity is infinite.

The problem for optimizing the transmission power and transferred energy for an $M$-horizon setting is the following:

$$
\begin{array}{ll}
\max _{p_{i, k}, T_{i, m}(k)} \sum_{k=1}^{M}\left\{\mathcal{D}_{1}\left(p_{i, k}\right)+\mathcal{D}_{2}\left(p_{i, k}\right)\right\} \\
\text { s.t. } & \frac{1}{M} \sum_{k=1}^{M} \frac{T-\tau_{k}}{T}\left(1-P_{d}\left(\tau_{k}\right)\right) \sum_{i=1}^{N} p_{i, k} g_{i, k} \leq Q_{a v g} \\
& 0 \leq p_{i, k} \leq P_{\max } ; \forall i, k, 0 \leq T_{i, m}(k), \forall i, m, k \\
& \sum_{r=1}^{k}\left(a_{i, r} p_{s} \tau_{r}+p_{i, r}\left(T-\tau_{r}\right)\right. \\
& \left.\left\{\left(1-P_{d}\left(\tau_{r}\right)\right) \mu+\left(1-P_{f a}\left(\tau_{r}\right)\right)(1-\mu)\right\}\right) \\
\leq & B_{i}+\sum_{r=1}^{k-1} H_{i, r}-\sum_{r=1}^{k} \sum_{m \in \mathcal{N}_{T, i}} T_{i, m}(r) \\
& +\sum_{r=1}^{k-1} \sum_{m \in \mathcal{N}_{R, i}} \eta_{m, i} T_{m, i}(r) ; \forall i, k .
\end{array}
$$

Remark: In the objective function, $\mathcal{D}_{1}$ and $\mathcal{D}_{2}$ are defined in (7). The expectations from (12) and (13) are removed with respect to (8) and (9), because the random parameters such as channel gains and energy arrival process are known noncausally in this case.

We formulate the Lagrangian for the problem as following:

$$
\begin{aligned}
\mathcal{L} & \left.\left\{p_{i, k}\right\},\left\{T_{i, m}(k)\right\}, \lambda,\left\{\delta_{i, k}\right\},\left\{\alpha_{i, k}\right\},\left\{v_{i, m, k}\right\},\left\{\beta_{i, k}\right\}\right) \\
= & \sum_{k=1}^{M} \mathcal{D}_{1}\left(p_{i, k}\right)+\sum_{k=1}^{M} \mathcal{D}_{2}\left(p_{i, k}\right) \\
& -\lambda\left\{\frac{1}{M} \sum_{k=1}^{M} \frac{T-\tau_{k}}{T}\left(1-P_{d}\left(\tau_{k}\right)\right) \sum_{i=1}^{N} p_{i, k} g_{i, k}-Q_{a v g}\right\} \\
& +\sum_{k=1}^{M} \sum_{i=1}^{N} \delta_{i, k} p_{i, k}-\sum_{k=1}^{M} \sum_{i=1}^{N} \alpha_{i, k}\left(p_{i, k}-P_{m a x}\right) \\
+ & \sum_{k=1}^{M} \sum_{i=1}^{N} \sum_{m=1, m \neq i}^{N} v_{i, m, k} T_{i, m}(k)-\sum_{k=1}^{M} \sum_{i=1}^{N} \beta_{i, k} \\
& \times\left\{\sum _ { r = 1 } ^ { k } \left(a_{i, r} p_{s} \tau_{r}+p_{i, r}\left(T-\tau_{r}\right)\right.\right. \\
& \quad-B_{i}-\sum_{r=1}^{k-1} H_{i, r}+\sum_{r=1}^{k} \sum_{m \in \mathcal{N}_{T, i}} T_{i, m}(r) \\
& \left.\quad-\sum_{r=1}^{k-1} \sum_{m \in \mathcal{N}_{R, i}} \eta_{m, i} T_{m, i}(r)\right\}
\end{aligned}
$$

where $a_{i, k}$ satisfy the constraint in (11). $\lambda, \delta_{i, k}, \alpha_{i, k}, v_{i, m, k}$ and $\beta_{i, k}$ are the non-negative dual variables associated with the average interference constraint, transmission power lower and upper limits, transferred energy constraints and energy causality constraint respectively.

We define $\mathcal{C}$ as a set of $p_{i, k}$ and $T_{i, m}(k)$ 's which satisfy (13), (14) and (15). We also define the vector of Lagrange parameters as $\tilde{\lambda}=\left(\lambda,\left\{\delta_{i, k}\right\},\left\{\alpha_{i, k}\right\},\left\{v_{i, m, k}\right\},\left\{\beta_{i, k}\right\}\right)$. Then the Lagrange dual function is expressed as:

$$
\begin{aligned}
& g(\tilde{\lambda})=\max _{\left\{p_{i, k}, T_{i, m}(k)\right\} \in \mathcal{C}} \mathcal{L}\left(\left\{p_{i, k}\right\},\left\{T_{i, m}(k)\right\}, \lambda,\left\{\delta_{i, k}\right\},\right. \\
& \left.\left\{\alpha_{i, k}\right\},\left\{v_{i, m, k}\right\},\left\{\beta_{i, k}\right\}\right)
\end{aligned}
$$

The dual problem is defined as:

$$
\min _{\tilde{\lambda} \succeq \mathbf{0}} g(\tilde{\lambda})
$$

where $\succeq$ denotes elementwise inequality. The above mentioned dual problem can be solved using a sub-gradient method [48]. We determine the dual function $g(\lambda)$ by solving the KKT conditions for the optimization problem, where the primary and dual solutions are denoted as $p_{i, k}^{\star}, \delta_{i, k}^{\star}, \alpha_{i, k}^{\star}, T_{i, m}^{\star}(k), v_{i, m, k}^{\star}, \beta_{i, k}^{\star}$. The optimality conditions are given as the following:

$\frac{\partial \mathcal{L}}{\partial p_{i, k}}=\chi_{1}+\chi_{2}+\delta_{i, k}^{\star}-\alpha_{i, k}^{\star}-\frac{T-\tau_{k}}{M T} \lambda g_{i, k}\left(1-P_{d}\left(\tau_{k}\right)\right)$ 


$$
\begin{aligned}
& \times\left(T-\tau_{k}\right)\left(\left(1-P_{d}\left(\tau_{k}\right)\right) \mu+\left(1-P_{f a}\left(\tau_{k}\right)\right)(1-\mu)\right) \\
& \quad \times \sum_{r=k}^{M} \beta_{i, r}^{\star}=0 \forall i, k \\
& \frac{\partial \mathcal{L}}{\partial T_{i, m}(k)}=0 \forall i, m, k \\
& \lambda\left\{\frac{1}{M} \sum_{k=1}^{M} \frac{T-\tau_{k}}{T}\left(1-P_{d}\left(\tau_{k}\right)\right) \sum_{i=1}^{N} p_{i, k} g_{i, k}-Q_{a v g}\right\}=0 \\
& \delta_{i, k}^{\star} p_{i, k}^{\star}=0 \forall i, k ; \quad v_{i, m, k}^{\star} T_{i, m}^{\star}(k)=0 \forall i, m, k \\
& \alpha_{i, k}^{\star}\left(p_{i, k}^{\star}-P_{\max }\right)=0 \forall i, k \\
& \tilde{\lambda} \succeq \mathbf{0}, \quad(13),(14),(15) \text { hold. }
\end{aligned}
$$

where $\chi_{1}, \chi_{2}$ represents the gradients of the achievable sum rate with respect to $p_{i, k}$ when the $\mathrm{PU}$ is active and idle, respectively. The expression for $\chi_{1}$ and $\chi_{2}$ can be written as:

$$
\begin{aligned}
& \chi_{1}=\frac{\mathrm{d} \mathcal{D}_{1}}{p_{i, k}}=\frac{\mu\left(T-\tau_{k}\right)}{M T \log 2} \frac{h_{i, k}\left(1-P_{d}\left(\tau_{k}\right)\right)}{\sigma^{2}+\sigma_{i n}^{2}+\sum_{l=1}^{k} p_{l, k}^{\star} h_{l, k}} \\
& \chi_{2}=\frac{\mathrm{d} \mathcal{D}_{2}}{p_{i, k}}=\frac{(1-\mu)\left(T-\tau_{k}\right)}{M T \log 2} \frac{h_{i, k}\left(1-P_{f a}\left(\tau_{k}\right)\right)}{\sigma^{2}+\sum_{l=1}^{k} p_{l, k}^{\star} h_{l, k}} .
\end{aligned}
$$

a) Optimal transmission power: From the above mentioned KKT conditions we find $p_{i, k}^{\star}$ by following the same approach as [19]. To derive $p_{i, k}^{\star}$ we propose the following lemma:

Lemma 1: Let $i$ and $j$ be two arbitrary SUs, where $i, j \in$ $\{1,2, \ldots, N\}$ with $p_{i, k}^{\star}>0$ and $p_{j, k}^{\star}=0$, then the following must be satisfied: $\frac{d_{i, k}}{\lambda e_{i, k}+f_{i, k}} \geq \frac{d_{j, k}}{\lambda e_{j, k}+f_{j, k}} \forall k$, where $d_{i, k}, e_{i, k}, f_{i, k}$ are given by the following expressions:

$$
\begin{aligned}
d_{i, k}= & \frac{T-\tau_{k}}{M T \log 2} h_{i, k} ; e_{i, k}=\frac{T-\tau_{k}}{M T \log 2} g_{i, k}\left(1-P_{d}\left(\tau_{k}\right)\right) \\
f_{i, k}= & \left(T-\tau_{k}\right)\left(\left(1-P_{d}\left(\tau_{k}\right)\right) \mu+\left(1-P_{f a}\left(\tau_{k}\right)\right)(1-\mu)\right) \\
& \times \sum_{r=k}^{M} \beta_{i, r}^{\star} .
\end{aligned}
$$

Proof: Please refer to Appendix A.

Now let $\pi$ be a permutation over the set $\{1,2, \ldots, N\}$ such that $\frac{d_{\pi(i), k}}{\lambda e_{\pi(i), k}+f_{\pi(i), k}} \geq \frac{d_{\pi(j), k}}{\lambda e_{\pi(j), k}+f_{\pi(j), k}}$ when $i<j$, $i, j \in\{1,2, \ldots, N\}$. Suppose that there are $|\mathcal{I}|$ SUs that can transmit where $\mathcal{I} \subseteq\{1,2, \ldots, N\}$. It can be checked that $\mathcal{I}=\{\pi(1), \pi(2), \ldots, \pi(|\mathcal{I}|)\}$. The following lemma provides a way to determine $|\mathcal{I}|$.

Lemma 2: $|\mathcal{I}|$ is given by the largest value of $x$ such that:

$$
\begin{aligned}
\frac{d_{\pi(x), k}}{\lambda e_{\pi(x), k}+f_{\pi(x), k}}>\left\{\frac{\mu\left(1-P_{d}\left(\tau_{k}\right)\right)}{\sigma^{2}+\sigma_{i n}^{2}+\sum_{y=1}^{x-1} P_{\max } h_{\pi(y), k}}\right. \\
\left.+\frac{(1-\mu)\left(1-P_{f a}\left(\tau_{k}\right)\right)}{\sigma^{2}+\sum_{y=1}^{x-1} P_{\max } h_{\pi(y), k}}\right\}^{-1}
\end{aligned}
$$

and the optimal transmission power for the $\pi(\mathcal{I})^{t h}$ SU can be expressed as $p_{\pi(|\mathcal{I}|), k}^{\star}=\min \left\{P_{\max }, P_{k}^{*}\right\}$, whereas $p_{\pi(a), k}^{\star}=P_{\max }, a<|\mathcal{I}|$ and $p_{\pi(a), k}^{\star}=0, a>|\mathcal{I}|$, and $P_{k}^{*}$ is the solution to the nonlinear (quadratic) equation (43) (see Appendix B).

Proof: Please refer to Appendix B. b) Optimal transferred energy: The KKT conditions corresponding to $T_{i, m}(k)$ are as follows:

$$
\frac{\partial \mathcal{L}}{\partial T_{i, m}(k)}\left\{\begin{array}{lll}
\geq 0 & \text { for } & T_{i, m}^{\star}(k)=0 \\
=0 & \text { for } & T_{i, m}^{\star}(k)>0
\end{array}\right.
$$

The necessary condition for the energy transfer is summarized in the following lemma.

Lemma 4: If it is optimal to transfer energy from the $i^{\text {th }}$ $\mathrm{SU}$ to the $m^{\text {th }} \mathrm{SU}$ at time slot $k$, i.e., $T_{i, m}^{\star}(k)>0$, then $\nu_{m, k+1}=\eta_{i, m} \nu_{i, k}$, where $\nu_{m, k}$ is the inverted sum of the future Lagrangian multipliers and expressed as : $\nu_{m, k}=$ $\left\{\sum_{r=k}^{M} \beta_{m, r}\right\}^{-1}$.

Proof: As according to the KKT condition (22), we know that if $T_{i, m}^{\star}(k)>0$ then $\frac{\partial \mathcal{L}}{\partial T_{i, m}(k)}=0$, and $v_{i, m, k}^{\star}=0$. Thus, we have $\sum_{r=k}^{M} \beta_{i, r}^{\star}=\eta_{i, m} \sum_{r=k+1}^{M} \beta_{m, r}^{\star}$, which leads to the required result.

Similar to [25], the above result can be used to show that energy transfer in both directions between a pair of SUs at the same time slot cannot be an optimal since the efficiency factor $\eta_{i, m} \leq 1$.

2) Optimal Sensing Time: Fixing the transmission power and the transferred energy would result in the optimization problem:

$$
\begin{aligned}
& \max _{\left\{\tau_{k}\right\} \in(0, T)} \sum_{k=1}^{M}\left\{\mathcal{D}_{1}\left(\tau_{k}\right)+\mathcal{D}_{2}\left(\tau_{k}\right)\right\} \\
& \text { s.t. } \frac{1}{M} \sum_{k=1}^{M} \frac{T-\tau_{k}}{T}\left(1-P_{d}\left(\tau_{k}\right)\right) \sum_{i=1}^{N} p_{i, k} g_{i, k} \leq Q_{a v g} \\
& \sum_{r=1}^{k}\left(a_{i, r} p_{s} \tau_{r}+p_{i, r}\left(T-\tau_{r}\right)\left(\left(1-P_{d}\left(\tau_{r}\right)\right) \mu\right.\right. \\
& \left.\left.\quad+\left(1-P_{f a}\left(\tau_{r}\right)\right)(1-\mu)\right)\right) \leq B_{i}+\sum_{r=1}^{k-1} H_{i, r} \\
& -\sum_{r=1}^{k} \sum_{m \in \mathcal{N}_{T, i}} T_{i, m}(r)+\sum_{r=1}^{k-1} \sum_{m \in \mathcal{N}_{R, i}} \eta_{m, i} T_{m, i}(r), 0 \leq \tau_{k} \leq T
\end{aligned}
$$

where the lower bound on $\tau_{k}$ is changed to $\tau_{l}$ in case of the heuristic policy for determining $a_{i, k}$.

We formulate the Lagrangian for the problem as follows:

$$
\begin{aligned}
& \mathcal{L}=\sum_{k=1}^{M} \mathcal{D}_{1}\left(\tau_{k}\right)+ \sum_{k=1}^{M} \mathcal{D}_{2}\left(\tau_{k}\right) \\
&-\lambda\left(\frac{1}{M} \sum_{k=1}^{M}\right.\left.\frac{T-\tau_{k}}{T}\left(1-P_{d}\left(\tau_{k}\right)\right) \sum_{i=1}^{N} p_{i, k} g_{i, k}-Q_{a v g}\right) \\
&-\sum_{k=1}^{M} \sum_{i=1}^{N} \beta_{i, k}\left(\sum _ { r = 1 } ^ { k } \left(a_{i, r} p_{s} \tau_{r}+p_{i, r}\left(T-\tau_{r}\right)\left(\left(1-P_{d}\left(\tau_{r}\right)\right) \mu\right.\right.\right. \\
&\left.\left.+\left(1-P_{f a}\left(\tau_{r}\right)\right)(1-\mu)\right)\right)-B_{i}-\sum_{r=1}^{k-1} H_{i, r} \\
&\left.+\sum_{r=1}^{k} \sum_{m \in \mathcal{N}_{T, i}} T_{i, m}(r)-\sum_{r=1}^{k-1} \sum_{m \in \mathcal{N}_{R, i}} \eta_{m, i} T_{m, i}(r)\right)
\end{aligned}
$$


The gradient of the Lagrangian with respect to $\tau_{k}$ can be written as:

$$
\begin{aligned}
\frac{\partial \mathcal{L}}{\partial \tau_{k}}= & \left(\lambda \omega_{k}^{(3)}-\omega_{k}^{1}\right)\left(1-P_{d}\right)-\omega_{k}^{(2)}\left(1-P_{f a}\right)-\omega_{k}^{(4)} \\
& +\left(\lambda \omega_{k}^{(3)}+\omega_{k}^{5}-\omega_{k}^{(1)}\right)\left(T-\tau_{k}\right) \frac{\partial P_{d}}{\partial \tau_{k}} \\
& +\left(\omega_{k}^{(6)}-\omega_{k}^{(2)}\right)\left(T-\tau_{k}\right) \frac{\partial P_{f a}}{\partial \tau_{k}}
\end{aligned}
$$

where $\omega_{k}^{(1)}, \omega_{k}^{(2)} \omega_{k}^{(3)} \omega_{k}^{(4)} \omega_{k}^{(5)} \omega_{k}^{(6)}$ are given by the following expressions:

$$
\begin{aligned}
\omega_{k}^{(1)}= & \frac{\mu}{M T} \log \left\{1+\frac{\sum_{i=1}^{N} p_{i, k} h_{i, k}}{\sigma^{2}+\sigma_{i n}^{2}}\right\} \\
\omega_{k}^{(2)}= & \frac{(1-\mu)}{M T} \log \left\{1+\frac{\sum_{i=1}^{N} p_{i, k} h_{i, k}}{\sigma^{2}}\right\} \\
\omega_{k}^{(3)}= & \frac{1}{M T} \sum_{i=1}^{N} p_{i, k} g_{i, k} \\
\omega_{k}^{(4)}= & \sum_{i=1}^{N}\left(a_{i, k} p_{s}-p_{i, k}\left(\left(1-P_{d}\right) \mu+\left(1-P_{f a}\right)(1-\mu)\right)\right) \\
& \times \sum_{r=k}^{M} \beta_{i, r} \\
\omega_{k}^{(5)}= & \sum_{i=1}^{N} \mu p_{i, k} \sum_{r=k}^{M} \beta_{i, r} ; \omega_{k}^{(6)}=\sum_{i=1}^{N}(1-\mu) p_{i, k} \sum_{r=k}^{M} \beta_{i, r}
\end{aligned}
$$

This optimization problem is not convex in $\tau_{k}$ and a locally optimum solution for the sensing time can be found by solving the necessary KKT conditions $\frac{\partial \mathcal{L}}{\partial \tau_{k}}=0$ for $0<\tau_{k}<T$, while $\frac{\partial \mathcal{L}}{\partial \tau_{k}} \geq 0$ for $\tau_{k}=T$ and $\frac{\partial \mathcal{L}}{\partial \tau_{k}} \leq 0$ for $\tau_{k}=0$ or $\tau_{k}=\tau_{l}$.

The feasibility of obtaining a locally optimal $\tau_{k}$ is guaranteed if the following conditions are satisfied:

$$
\begin{aligned}
& \left.\frac{\partial \mathcal{L}}{\partial \tau_{k}}\right|_{\tau_{k} \rightarrow T}=\left(\lambda \omega_{k}^{(3)}-\omega_{k}^{1}\right)\left(1-P_{d}\right) \\
& -\omega_{k}^{(2)}\left(1-P_{f a}\right)-\omega_{k}^{(4)}>0 \\
& \left.\frac{\partial \mathcal{L}}{\partial \tau_{k}}\right|_{\tau_{k} \rightarrow 0}<0
\end{aligned}
$$

For a given set of parameters defining the optimization problem, one needs to check whether there is a set of Lagrange parameters $\lambda, \beta_{i, r}$ that satisfy the above mentioned conditions. To this end, one can build up a lookup table of the relevant Lagrange parameters for given sets of optimization problem parameters (using numerical methods as no direct closed form relationship exists), and use this for a feasibility check.

If feasible, the equation $\frac{\partial \mathcal{L}}{\partial \tau_{k}}=0$ can be solved by applying a bisection search method in the interval $0 \leq \tau_{k} \leq T$ or $\tau_{l} \leq \tau_{k} \leq T$, depending on the method for finding $a_{i, k}$.
Algorithm 1 Algorithm for Calculation of Optimal Parameters for Fixed $a_{i, k}$

1: Initialization: Choose a feasible initial sensing time $\tau_{k}^{0}$ for all $k \in\{1,2, \ldots, M\}$, tolerance of the algorithm $\delta$, Lagrange parameter $\lambda^{0}$ and step-size of the sub-gradient algorithm $\epsilon$. Choose also the initial achievable sum rate as 0 .

2: repeat

3: $\quad$ For $l=0,1, \ldots(l$ is an iteration number $)$

4: Determine the optimal transmission power $p_{i, k}^{l+1}$ by solving the quadratic non-linear equation (43).

5: Compute the shared energy $T_{i, m}^{l+1}(k)$ by solving the following iteration:

$$
T_{i, m}^{l+1}(k)=\left\{T_{i, m}^{l}(k)-\epsilon\left(\sum_{r=k}^{M} \beta_{i, r}^{l}-\eta_{i, m} \sum_{r=k+1}^{M} \beta_{m, r}^{l}\right)\right\}^{+}
$$

where $\beta_{i, k}$ 's are determined by a dual sub-gradient algorithm.

6: $\quad$ Fixing the $p_{i, k}^{l+1}$ and $T_{i, k}^{l+1}$, the $\tau_{k}^{l+1}$ is solved by applying a bisection search method.

7: Solve for $\lambda$ in the optimization problem (18) by a dual subgradient method given as

$$
\begin{aligned}
& \lambda^{l+1}=\left\{\lambda^{l}-\epsilon\left\{Q_{a v g}-\mathrm{E}\left\{\sum_{k=1}^{M} \frac{T-\tau_{k}^{l+1}}{M T}(1-\right.\right.\right. \\
& \left.\left.\left.\left.P_{d}\left(\tau_{k}^{l+1}\right)\right) \sum_{i=1}^{N} p_{i, k}^{l+1} g_{i, k}\right\}\right\}\right\}^{+} .
\end{aligned}
$$

8: until Convergence $\left|\mathcal{C}\left(p_{i, k}^{l+1}, \tau_{k}^{l+1}\right)-\mathcal{C}\left(p_{i, k}^{l}, \tau_{k}^{l}\right)\right| \leq \delta \quad \forall i, m, k$, where $\mathcal{C}\left(p_{i, k}, \tau_{k}\right)$ is the achievable sum rate expression from (8).

\section{B. Algorithm for Locally Optimal Transmission Power, Shared Energy and Sensing Time}

In this section we summarize the above mentioned optimization procedure in an algorithmic form (Algorithm 1) for computing locally optimal values of the transmission power, sensing time and shared energy. We assume that there are pre-decided tolerance values $\epsilon$ and $\delta . \mathcal{C}\left(p_{i, k}, \tau_{k}\right)$ represents the achievable sum rate corresponding to the transmission power $p_{i, k}$ and sensing time $\tau_{k}$.

Convergence analysis: The objective function of the optimization problem in (8) is non-decreasing from iteration to iteration of the alternating optimization procedure as at each iteration it is maximized with respect to a parameter by fixing the other parameters. It can be easily shown that by considering only the short term constraints (10), in the extreme scenario, i.e., $a_{i, k}=1, \forall i, k, \forall k, \tau_{k}^{\star}=0, \forall k$ and $p_{i, k}^{\star}=P_{\text {max }}, \forall i, k$, the achievable sum rate is upper bounded by the following expression:

$$
\begin{aligned}
& \mathrm{E}\left\{\sum_{k=1}^{M} \frac{\mu}{2 M} \log _{2}\left\{1+\frac{\sum_{i=1}^{N} P_{\max } h_{i, k}}{\sigma^{2}+\sigma_{i n}^{2}}\right\}\right. \\
& \left.\quad+\sum_{k=1}^{M} \frac{(1-\mu)}{2 M} \log _{2}\left\{1+\frac{\sum_{i=1}^{N} P_{\max } h_{i, k}}{\sigma^{2}}\right\}\right\}
\end{aligned}
$$

where the expectation is taken over $h_{i, k}$. Note that the additional constraints can only limit the feasible set further and thus lower the objective function value. Using Jensen's 
inequality, we can write:

$$
\begin{aligned}
\mathrm{E}\left\{\sum_{k=1}^{M} \frac{\mu}{2 M} \log _{2}\left\{1+\frac{\sum_{i=1}^{N} P_{\max } h_{i, k}}{\sigma^{2}+\sigma_{i n}^{2}}\right\}\right. \\
\left.+\sum_{k=1}^{M} \frac{(1-\mu)}{2 M} \log _{2}\left\{1+\frac{\sum_{i=1}^{N} P_{\max } h_{i, k}}{\sigma^{2}}\right\}\right\} \\
\leq \sum_{k=1}^{M} \frac{\mu}{2 M} \log _{2}\left\{1+\frac{\sum_{i=1}^{N} P_{\max } \mathrm{E}\left\{h_{i, k}\right\}}{\sigma^{2}+\sigma_{i n}^{2}}\right\} \\
\quad+\sum_{k=1}^{M} \frac{(1-\mu)}{2 M} \log _{2}\left\{1+\frac{\sum_{i=1}^{N} P_{\max } \mathrm{E}\left\{h_{i, k}\right\}}{\sigma^{2}}\right\} \\
=\sum_{k=1}^{M} \frac{\mu}{2 M} \log _{2}\left\{1+\frac{\sum_{i=1}^{N} P_{\max }}{\sigma^{2}+\sigma_{i n}^{2}}\right\} \\
\quad+\sum_{k=1}^{M} \frac{(1-\mu)}{2 M} \log _{2}\left\{1+\frac{\sum_{i=1}^{N} P_{\max }}{\sigma^{2}}\right\}
\end{aligned}
$$

Thus, the objective function is upper bounded by the achievable sum rate using a feasible policy involving $a_{i, k}, p_{i, k}, \tau_{k}$. Since a non-decreasing sequence that is upper bounded must converge, it follows that the alternating optimization algorithm above converges to a local optimum.

\section{Non-Adaptive Optimization With Non-Causal CSI}

In this section we propose a policy (albeit sub-optimal) where instead of optimizing the sensing time for each individual time slot, we use a constant (but optimized) sensing time for all time slots. In this case, the analysis for the transmission power and transferred energy (for a fixed sensing time) remains the same as before. A locally optimum nonadaptive sensing time can be found by solving the following optimization problem (where $p_{i, k}, T_{i, m}(k)$ are kept fixed):

$$
\begin{aligned}
\max _{\{\tau\}} & \sum_{k=1}^{M}\left\{\mathcal{D}_{1}(\tau)+\mathcal{D}_{2}(\tau)\right\} \\
\text { s.t. } & \frac{1}{M} \sum_{k=1}^{M} \frac{T-\tau}{T}\left(1-P_{d}(\tau)\right) \sum_{i=1}^{N} p_{i, k} g_{i, k} a_{i, k} \leq Q_{a v g} \\
& 0 \leq \tau \leq T \\
& \sum_{r=1}^{k}\left(a_{i, r} p_{s} \tau+p_{i, r}(T-\tau)\left(\left(1-P_{d}(\tau)\right) \mu\right.\right. \\
& \left.\left.\quad+\left(1-P_{f a}(\tau)\right)(1-\mu)\right)\right) \leq B_{i}+\sum_{r=1}^{k-1} H_{i, r} \\
& -\sum_{r=1}^{k} \sum_{m \in \mathcal{N}_{T, i}} T_{i, m}(r)+\sum_{r=1}^{k-1} \sum_{m \in \mathcal{N}_{R, i}} \eta_{m, i} T_{m, i}(r)
\end{aligned}
$$

We can formulate a Lagrangian $\mathcal{L}_{\infty}$ similar to (24), and solve for a locally optimum $\tau^{*}$ by solving $\frac{\partial \mathcal{L}_{\infty}}{\partial \tau}=0$. Feasibility and convergence analysis of this setting can be carried out in a similar fashion to the adaptive case.

\section{Causal Optimization With Finite Battery}

In this section, we consider the finite horizon achievable sum rate optimization problem under the more realistic assumption of causal CSI and ESI, and a finite battery setting. Similar to the previous section, this optimization can be done for non-adaptive and adaptive sensing time both. For space limitations, we only consider the adaptive sensing time strategy below.

\section{A. Achievable Sum Rate Optimization With Causal CSI and ESI}

The throughput optimization in the context of causal CSI and ESI with finite battery is a stochastic control problem and can be solved by dynamic programming (DP) techniques. Note that in the case of DP, at each stage, it is natural to consider the optimization over $a_{i, k}$ in an exhaustive search, along with optimization over discretized values of all the continuous optimization variables for facilitating numerical implementation.

1) Information Pattern: In each time slot the FC receives the CSI between the PU transmitter and the SU receivers $\mathbf{g}_{k}=\left\{g_{1, k}, g_{2, k}, \ldots, g_{N, k}\right\}$ causally with the assumption of a priori knowledge of the PU transmission levels and channel reciprocity. The CSI between the SU transmitter and the FC $\mathbf{h}_{k}=\left\{h_{1, k}, h_{2, k}, \ldots, h_{N, k}\right\}$ is also received at the FC by channel training, estimation and feedback mechanisms. The battery state information of the individual SUs are collected at the FC and collated as $\mathbf{B}_{k}=B_{1, k}, B_{2, k}, \ldots, B_{N, k}$. The information available at the $k^{t h}$ time slot is given by the tuple $\mathbf{J}_{k}=\left\{\mathbf{g}_{k}, \mathbf{h}_{k}, \mathbf{B}_{k}, \mathbf{J}_{k-1}\right\}$.

2) Dynamic Programming Algorithm: We denote the immediate per-stage cost function for the stochastic control problem as

$$
\begin{aligned}
\mathcal{C}\left(p_{i, k}, \tau_{k}\right)= & \mathcal{D}_{1}\left(p_{i, k}, \tau_{k}\right)+\mathcal{D}_{2}\left(p_{i, k}, \tau_{k}\right) \\
& -\lambda\left\{\frac{T-\tau_{k}}{M T}\left(1-P_{d}\left(\tau_{k}\right)\right) \sum_{i=1}^{N} p_{i, k} g_{i, k}-Q_{a v g}\right\}
\end{aligned}
$$

Here, $\lambda$ is the Lagrange parameter corresponding to the average interference constraint. $\mathcal{D}_{1}$ and $\mathcal{D}_{2}$ are defined in (7).

Combining all the other short term constraints on the transmission power, shared energy and sensing time, and the energy causality constraint, along with the binary search space of $a_{i, k}$, we define the feasible set for the optimization variables as:

$$
\begin{aligned}
\mathcal{A}=\{ & \left(a_{i, k}, p_{i, k}, T_{i, m}(k), \tau_{k}\right): a_{i, k}, p_{i, k}, T_{i, m}(k), \tau_{k} \\
& \text { satisfy }(10),(11)\}
\end{aligned}
$$

For a fixed $\lambda$, the optimal values of transmission power, sensing time and energy shared can be determined by the following theorem:

Theorem 1: If the initial condition is $\mathbf{J}_{1}=\left\{\mathbf{g}_{1}, \mathbf{h}_{1}, \mathbf{B}_{1}\right\}$, then the value of the finite horizon finite battery problem with causal information is given by $V_{1}\left(\mathbf{g}_{1}, \mathbf{h}_{1}, \mathbf{B}_{1}\right)$, which can be computed by the backward Bellman dynamic programming 
equation:

$$
\begin{aligned}
& V_{k}\left(\mathbf{g}_{k}, \mathbf{h}_{k}, \mathbf{B}_{k}\right)=\max _{\left(a_{i, k}, p_{i, k}, T_{i, m}(k), \tau_{k}\right) \in \mathcal{A}} \\
& \times\left[C\left(p_{i, k}, \tau_{k}\right)+\mathbf{E}\left[V_{k+1}\left(\mathbf{g}_{k+1}, \mathbf{h}_{k+1}, \mathbf{B}_{k+1}\right) \mid a_{i, k}, p_{i, k},\right.\right. \\
& \left.\left.T_{i, m}(k), \tau_{k}\right]\right], k=0,1, \ldots, M-1
\end{aligned}
$$

where the terminal condition is given by $V_{M}\left(\mathbf{g}_{M}, \mathbf{h}_{M}, \mathbf{B}_{M}\right)$ is given as the solution to the optimization problem (8)-(11) for a single slot $k=M$ with the causal knowledge of $\mathbf{g}_{M}, \mathbf{h}_{M}, \mathbf{B}_{M}$.

Proof: The proof can be obtained by the standard optimality conditions for the finite horizon stochastic control problem [49], and is omitted for brevity.

The solution of the causal optimization problem, which can be computed numerically, is obtained as:

$$
\begin{gathered}
\left\{a_{i, k}^{\star}, p_{i, k}^{\star}, T_{i, m}^{\star}(k), \tau_{k}^{\star}\right\}=\operatorname{argmax}_{a_{i, k}, p_{i, k}, T_{i, m}(k), \tau_{k} \in \mathcal{A}} \\
\times\left[C\left(p_{i, k}, \tau_{k}\right)+\mathbf{E}\left[V_{k+1}\left(\mathbf{g}_{k+1}, \mathbf{h}_{k+1}, \mathbf{B}_{k+1}\right) \mid a_{i, k},\right.\right. \\
\left.\left.p_{i, k}, T_{i, m}(k), \tau_{k}\right]\right] .
\end{gathered}
$$

3) Sub-Gradient Method for the Lagrange Parameters: The optimal value of $\lambda$ from (29) can be found by solving the following equation:

$$
\lambda\left\{\mathbf{E}\left\{\sum_{k=1}^{M} \frac{T-\tau_{k}}{M T}\left(1-P_{d}\left(\tau_{k}\right)\right) \sum_{i=1}^{N} p_{i, k} g_{i, k}\right\}-Q_{a v g}\right\}=0
$$

This is achieved by using a sub-gradient algorithm [36] by updating the value of $\lambda$

$$
\begin{aligned}
\lambda^{l+1}=\left\{\lambda^{l}-\alpha^{l}\left\{Q_{a v g}-\mathrm{E}\right.\right. & \left\{\sum_{k=1}^{M} \frac{T-\tau_{k}}{M T}\left(1-P_{d}\left(\tau_{k}\right)\right)\right. \\
& \left.\left.\left.\times \sum_{i=1}^{N} p_{i, k} g_{i, k}\right\}\right\}\right\}^{+}
\end{aligned}
$$

where $l$ is the iteration index and $\alpha^{l}$ is a scalar step size parameter for the $l^{\text {th }}$ iteration satisfying $\sum_{l=1}^{\infty} \alpha^{l}=\infty$ and $\sum_{l=1}^{\infty}\left(\alpha^{l}\right)^{2}<\infty$, and $\{x\}^{+}=\max \{x, 0\}$.

Note that the DP algorithm (31) and the subgradient based update (33) are performed offline, iteratively until convergence, purely based on the statistics of the channel gains and harvested energy information. Since there is no closed form expression to the optimal solutions, one needs to discretize the search space for the continuous control variables $p_{i, k}, T_{i, m}(k)$ and $\tau_{k}$, and the state variables $\mathbf{g}_{k}, \mathbf{h}_{k}, \mathbf{B}_{k}$. With these discretized search space, the FC creates a lookup table which is shared with all SUs. In real time the FC receives the continuous valued channel gains and battery states and check for the closest quantization point in its lookup table. The table index for the optimal sensing time, transmission power and energy shared is fetched from the look-up table and sent to the individual SUs, and then used by the SUs for sensing, information transmission and energy sharing.

\section{Sub-Optimal Policies}

In order to apply the dynamic programming algorithm for a fixed value of $\lambda$, we discretize both the state space parameters $\mathbf{g}_{k}, \mathbf{h}_{k}, \mathbf{B}_{k}$ and action space parameters $p_{i, k}, T_{i, m}(k), \tau_{k}$, where $i=1,2, \ldots, N$ and $k=1,2, \ldots, M$. If we consider $\mathcal{A}$ and $\mathcal{S}$ to be the sets of discretized action (control) space and state space respectively for a given time slot (considering all the users together), then the complexity of the dynamic programming algorithm becomes $\mathcal{O}\left(\left(2^{N}|\mathcal{A}||\mathcal{S}|\right)^{M}\right)$, where the factor $2^{N}$ comes from the binary variable $a_{i, k}$ for $N$ secondary users. To combat this exponential complexity, in this section we propose a number of low-complexity suboptimal policies.

\section{A. Limited Look-Ahead Policy}

Here we propose a limited 2-Horizon look-ahead policy based on the approximate dynamic programming literature [39]. For this policy, only a two horizon lookup table in the backward Bellman algorithm is created. For any time slot, the causal optimization is performed with the help of the above mentioned lookup table considering only one future time slot at a time.

\section{B. Ad-Hoc Policy}

Here we formulate a sub-optimal policy based on the necessary conditions determined in Section IV for non-causal information with infinite battery and extend that approach to the causal information scenario with finite battery capacity. To alleviate the complexity associated with optimizing the achievable sum-rate over all time slots, we implement a greedy algorithm and formulate the optimization problem in terms of individual time slots. The optimization of the sensing time $\tau_{k}$ is done through a discretization process similar to the DP algorithm. The parameter corresponding to the decision to sense $a_{i, k}$ is determined using the heuristic policy proposed in Section III-B. The simplified optimization problem over individual time slots becomes:

$$
\begin{aligned}
& \max _{p_{i, k}} \frac{\mu}{M} \frac{T-\tau_{k}}{T}\left(1-P_{d}\left(\tau_{k}\right)\right) \log _{2}\left\{1+\frac{\sum_{i=1}^{N} p_{i, k} h_{i, k}}{\sigma^{2}+\sigma_{i n}^{2}}\right\} \\
& \quad+\frac{1-\mu}{M} \frac{T-\tau_{k}}{T}\left(1-P_{f a}\left(\tau_{k}\right)\right) \log _{2}\left\{1+\frac{\sum_{i=1}^{N} p_{i, k} h_{i, k}}{\sigma^{2}}\right\} \\
& \text { s.t. } \quad \frac{T-\tau_{k}}{T}\left(1-P_{d}\left(\tau_{k}\right)\right) \sum_{i=1}^{N} p_{i, k} g_{i, k} \leq Q_{a v g} \\
& 0 \leq p_{i, k} \leq P_{\max } \forall i, k ; 0 \leq \tau_{k} \leq T \forall k ; 0 \leq T_{i, m}(k) \forall i, m, k \\
& \quad\left(a_{i, k} p_{s} \tau_{k}+p_{i, k}\left(T-\tau_{k}\right)\right. \\
& \left.\quad \times\left\{\left(1-P_{d}\left(\tau_{k}\right)\right) \mu+\left(1-P_{f a}\left(\tau_{k}\right)\right)(1-\mu)\right\}\right) \leq B_{i, k}
\end{aligned}
$$

We can solve this problem using the same approach as in [19]. Using the techniques used in Section IV, we conclude the following:

- Only one SU in the set $\{1,2, \ldots, N\}$ would be able to transmit power from the open set $\left(0, P_{\max }\right)$. All the other SUs either transmit with $P_{\max }$ or do not transmit in the time slot under consideration.

- If $i \neq j$, and $p_{i, k}>0$ and $p_{j, k}=0$, then $\frac{h_{i, k}}{\lambda^{\star} \mu g_{i, k}+\delta_{i}^{*}} \geq$ $\frac{h_{i, k}}{\lambda^{\star} \mu g_{i, k}+\delta_{j}^{*}}$, where $\lambda^{\star}$ and $\delta_{i}^{*}$ are the optimal Lagrange parameters corresponding to (34) and (35), respectively.

- The above inequality also holds for the scenario when $p_{i, k}=P_{\max }$ and $p_{j, k} \in\left[0, P_{\max }\right)$. 
Thus we infer that for such choices of $i$ and $j$, the following condition holds:

$$
\left\{\frac{g_{j, k}}{h_{j, k}}-\frac{g_{i, k}}{h_{i, k}}\right\} \geq \frac{1}{\lambda^{\star} \mu}\left\{\frac{\delta_{i}^{\star}}{h_{i, k}}-\frac{\delta_{j}^{\star}}{h_{j, k}}\right\}
$$

If we fix $\lambda^{\star}>0$ and $\delta_{j}^{\star}=0$ then we can have $\frac{h_{i, k}}{g_{i, k}} \geq \frac{h_{j, k}}{g_{j, k}}$.

Using the above mentioned policy we sort all the SUs according to the descending order of the ratio of their direct and interference channel gains. Starting with the SU corresponding to the highest ratio, we determine the SU transmission power of the according to the following expression:

$$
\begin{aligned}
p_{i, k}^{\star} & =\min \left\{P_{\max }, \frac{T Q_{a v g}}{\left(T-\tau_{k}\right) g_{i, k}\left(1-P_{d}\left(\tau_{k}\right)\right)},\right. \\
& \left.\frac{B_{i, k}-p_{s} \tau_{k}}{\left(T-\tau_{k}\right)\left\{\left(1-P_{d}\left(\tau_{k}\right)\right) \mu+\left(1-P_{f a}\left(\tau_{k}\right)\right)(1-\mu)\right\}}\right\}
\end{aligned}
$$

If there is still energy left in the battery after the transmission process, the rest of the energy is shared to the SU with highest $\frac{\mu_{h}}{\mu_{q}}$, ensuring that there is no battery overflow [25]. The optimal transmission energy shared is given by the following expression:

$$
\begin{aligned}
T_{i, m}^{\star}(k)=\min \{\max \{ & \left\{B_{\max }-B_{m, k}+E_{m, k}\right. \\
& \left.\left.\left.-2 \mu_{H_{m}}\right\} / \eta_{i, m} ; 0\right\} ; B_{i, k}\right\} .
\end{aligned}
$$

\section{Computational Complexity}

Heuristic Policy for $a_{i, k}$ : The proposed heuristic policy for finding the spectrum access decision variable $a_{i, k}$ ensures that individual SUs can determine their corresponding spectrum access decision $a_{i, k}$ in a decentralized fashion. Compared to the optimal policy, no look-up table is required in the FC for specifically optimizing $a_{i, k}$ for the heuristic policy. Thus in this scenario, the value of $a_{i, k}$ can be instantaneously computed as the indicator variable $\mathcal{I}_{B_{i, k} \geq p_{s} \tau_{l}}$, resulting in a computational complexity of $\mathcal{O}(1)$ for a given $i, k$. However, for optimizing the rest of the optimization variables like $\tau_{k}$, $p_{i, k}$ and $T_{i, m}(k)$ look up table needs to be created and stored in FC as usual via dynamic programming, resulting in an exponential complexity of $\mathcal{O}\left((|\mathcal{A}||\mathcal{S}|)^{M}\right)$. Therefore the overall complexity of the heuristic algorithm is $\mathcal{O}\left(M N(|\mathcal{A}||\mathcal{S}|)^{M}\right)$.

Limited Look ahead Policy: For this sub-optimal policy, the look up table is only stored for two time slots. Thus the corresponding computational complexity is $\mathcal{O}\left(M\left(2^{N}|\mathcal{A}||\mathcal{S}|\right)^{2}\right)$.

Ad-hoc Policy: For the ad-hoc policy, the value of the transmission power $p_{i, k}, T_{i, m}(k)$ are obtained by the closed form expressions in (36-37), for each $k$, while $a_{i, k}$ is computed using the heuristic policy and $\tau_{k}$ is computed using a set of discretized values, say of cardinality $\left|A_{\tau}\right|$. In this case, the complexity is $\mathcal{O}\left(M N^{2}\left(\left|A_{\tau}\right||\mathcal{S}|\right)^{M}\right)$.

\section{Simulation Results}

In this section we present some numerical results to illustrate the performance of the various algorithms considered in

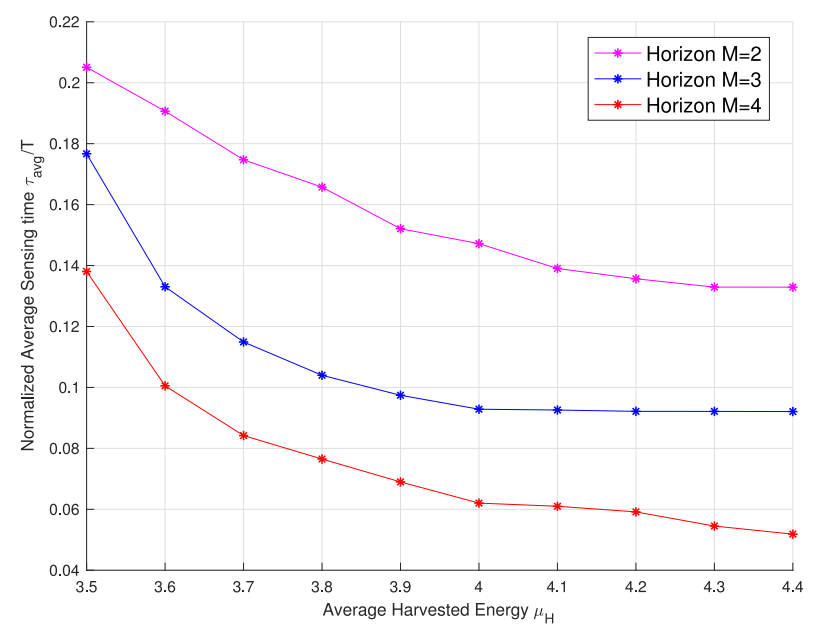

Fig. 3. Normalized average sensing time $\tau_{a v g}$ vs Average harvested energy $\mu_{H}$ with ratio of SU Tx-FC Rx and SU Tx-PU Rx average channel gain $\mu_{h} / \mu_{g}$ fixed.

the previous sections. We assume the energy harvesting process at the SUs is an exponentially distributed random process with a mean of $1 \mu \mathrm{J}$, unless otherwise stated. The PU activity probability is set to $\mu=0.8$ (we also present some results for $\mu=0.2$ later in this section). The sensing channel signal to noise ratio (SNR) is assumed to be $-15 \mathrm{~dB}$. The PU signal variance is taken to be $\sigma_{x}^{2}=1 \mathrm{~mW}$. The length of a time slot is taken as $T=2 \mathrm{~ms}$, and the individual mini-slot length is $0.001 \mathrm{~ms}$. The probability of false alarm limit of the heuristic policy $\kappa$ is taken to be 0.1 . The sampling frequency is assumed to be $1 \mathrm{MHz}$ and the normalized threshold of detection is assumed to be $\frac{\epsilon}{\sigma_{n}^{2}}=1.006$. This corresponds to a minimum sensing time limit $\tau_{l}=0.05 \mathrm{~ms}$. This constraint on the minimum sensing time has been applied only to the heuristic policy based methods.

We assume a secondary network with $N=2$ users and with a maximum transmission power of $10 \mathrm{~mW}$ each. The PU interference and noise variance for the channel between the SUs and the FC is taken to be $\sigma_{i n}^{2}=\sigma^{2}=4 \times 10^{-10} \mathrm{~W}$. Assuming the maximum average received SNR at the FC of approximately $5 \mathrm{~dB}$ (with the primary interference active), this corresponds to a path loss of approximately $-87 \mathrm{~dB}$ with a path loss exponent of 2.7 over a distance of 100 metres between the SU and the FC, using the simplified path loss model from [50, p. 46], where the reference distance for the antenna far field is $10 \mathrm{~m}$, and transmission frequency at $2.4 \mathrm{GHz}$. The energy transfer efficiency for energy sharing is assumed to be 0.4. The average interference limit (normalized by the path loss factor between the SUs and the PU receiver) $Q_{\text {avg }}=5 \mathrm{~mW}$. The initial battery level for each SU is assumed to be $0.4 \mu \mathrm{J}$. For the dynamic programming simulation the state space parameters $\mathbf{g}_{k}, \mathbf{h}_{k}, \mathbf{H}_{k}$ and the action space parameters $p_{i, k}, T_{i, m}(k), \tau_{k}$ are quantized into 5 different discrete levels.

In Fig. 3, we plot the average sensing time (averaged over the time horizon) for the non-causal CSI and ESI denoted by $\tau_{\text {avg }}$ with respect to the average harvested energy denoted by $\mu_{H}$, keeping the mean of the channel mean gains 


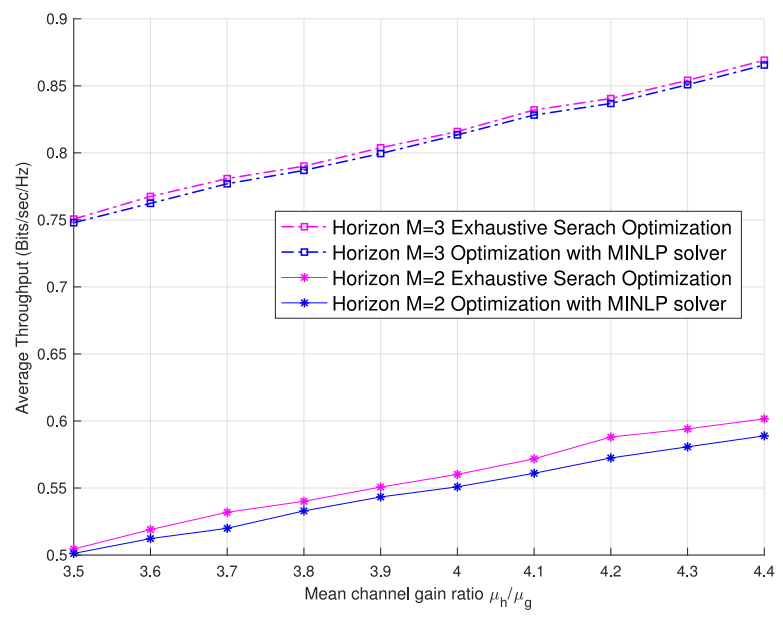

Fig. 4. Average Throughput vs ratio of SU Tx-FC Rx and SU Tx-PU Rx average channel gain $\mu_{h} / \mu_{g}$ with average harvested energy $\mu_{H}$ fixed with Exhaustive Search and MINLP solver.

denoted by $\mu_{g}$ and $\mu_{h}$ constant, for $M=2,3,4$. The averages are taken over 50 Monte-Carlo simulations. From Fig. 3, it is evident that the average sensing time $\tau_{\text {avg }}$ decreases monotonically by increasing the length of the horizon. As a numerical comparison, for mean harvested energy $\mu_{H}=3.5 \mu \mathrm{J}$, the normalized average sensing times corresponding to $M=3$ and $M=4$ are 12.1 and 31.7 percent less compared to $M=2$ respectively. This is due to the fact that increasing the length of horizon $M$ in the non-causal CSI and battery state scenario helps to spread out the sensing time over multiple time slots, which means on average the transmission time increases and the sensing time decreases with increasing $M$. A similar behaviour of $\tau_{\text {avg }}$ is observed when plotted against increasing $\mu_{h} / \mu_{g}$, keeping $\mu_{H}$ fixed, and hence not shown here.

Fig. 4 compares the average throughput with respect to the ratio of mean channel gain $\mu_{h} / \mu_{g}$ keeping $\mu_{H}$ fixed for horizon length $M=2$ and $M=3$ with non-causal information. The spectrum sensing decision variable is optimized using both the exhaustive search and a MINLP solver called MIDACO [51]. From the figure, it is noticeable that both approaches result in approximately the same throughput. Although the MINLP solver is computationally faster, neither of these methods is scalable for moderate to large values of $M, N$. Fig. 5 shows the average throughput plotted against the battery capacity $B_{\max }$ for the adaptive and the non-adaptive optimization policy with non-causal and causal information. These simulations are averaged over 100 Monte-Carlo iterations. We can notice that for $B_{\max }=1.4 \mu \mathrm{J}$ the average throughput corresponding to causal non-adaptive policy is 17.5 percent less compared to its adaptive counterpart. As expected, the adaptive policy outperforms its non-adaptive counterpart at the expense of higher computational complexity.

Fig. 6 shows the optimized average throughput, i.e., average throughput plotted against battery capacity $B_{\max }$ with non-causal CSI and battery state scenario with the exhaustive search technique (for the decision to sense), causal CSI and ESI with exhaustive search and heuristic policy (for the decision to sense). It is noticeable that for $M=4$ and

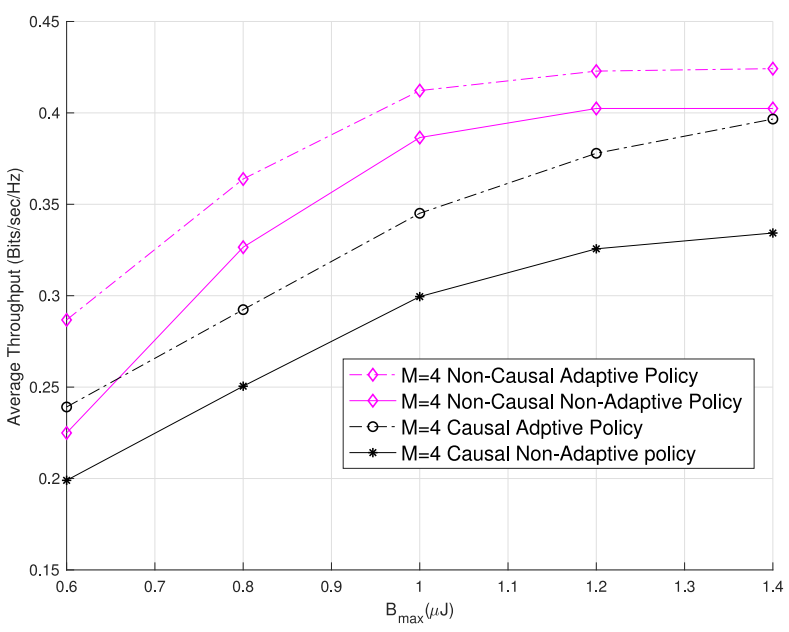

Fig. 5. Average Throughput vs Battery capacity $B_{\max }$ for Adaptive and Non-Adaptive Policy for Horizon length $\mathrm{M}=4$.

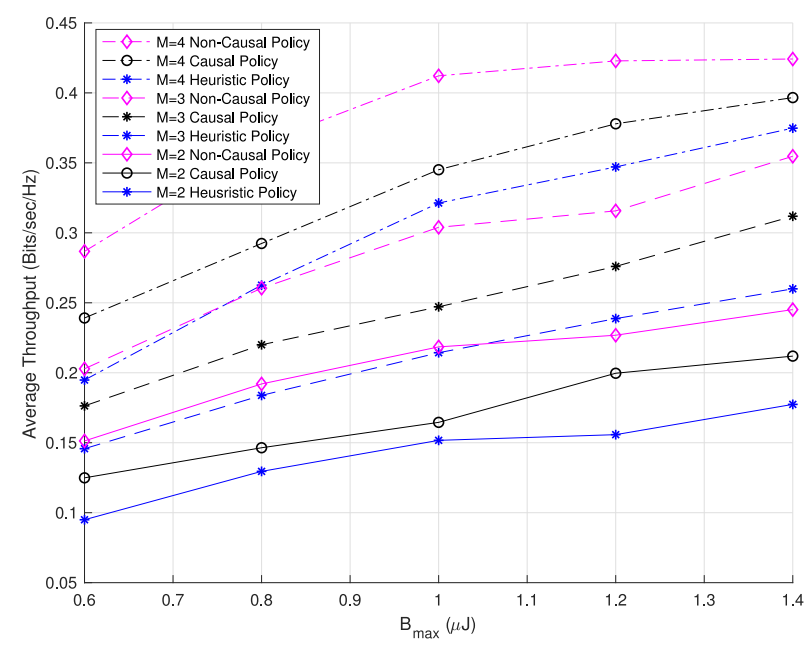

Fig. 6. Average Throughput vs Battery capacity $B_{\max }$ Non-Causal, Causal and Heuristic Policy.

$B_{\max }=1.4 \mu \mathrm{J}$ the average throughput corresponding to causal adaptive policy and heuristic policy are 5 and 11.7 percent less compared to its non-causal counterpart, respectively. As expected the average throughput increases with increasing horizon length and non-causal CSI/ESI scenario provides an upper bound for the causal counterpart. As the heuristic policy is less computationally complex, we are able to simulate the average sum throughput with causal information with this policy for horizon $M=10,15,20$ in Fig. 9. As expected, it is seen that the average sum throughput increases with increasing horizon length.

We have compared the policies proposed in our previous work in [39] with the ones introduced in this present work in Fig. 7. The figure includes plots for average throughput with respect to battery capacity $B_{\max }$ for Non-causal, causal and heuristic policy with horizon length $M=4$. The plots show that the policies introduced in this work outperforms their counterpart policies introduced in [39]. For a numerical comparison, for $B_{\max }=0.8 \mu \mathrm{J}$, the average throughput corresponding to the causal policy introduced in [39] is 13.3 percent 


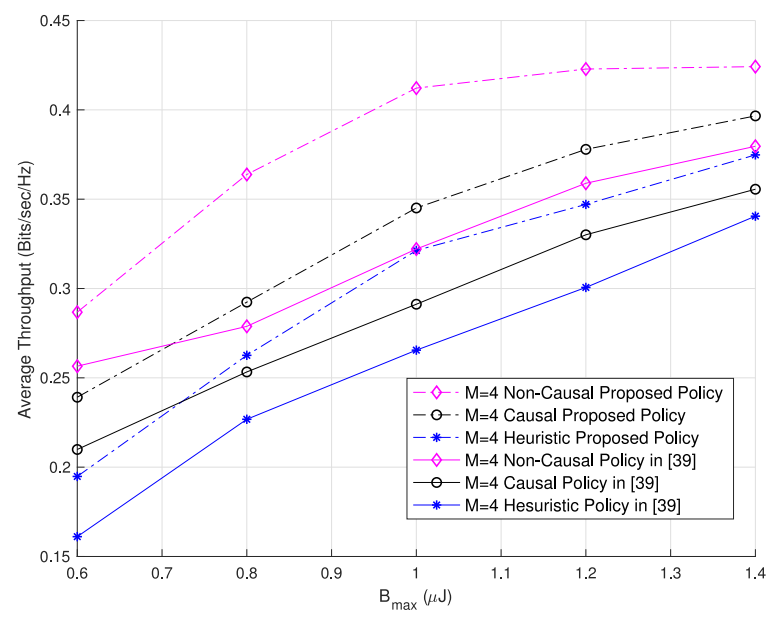

Fig. 7. Average Throughput comparison between Proposed policy and the policy in [38] for Non-Causal, Causal and Heuristic Policy vs Battery Capacity $B_{\max }$ for Horizon length $\mathrm{M}=4$.

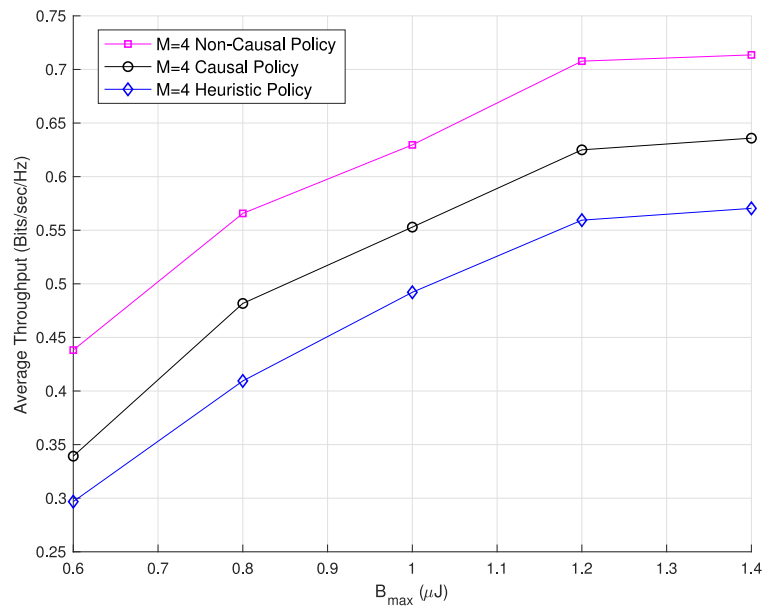

Fig. 8. Average throughput for Non-Causal, Causal and Heuristic Policy vs Battery capacity $B_{\max }$ for Horizon length $\mathrm{M}=4$ and PU activity probability $\mu=0.2$.

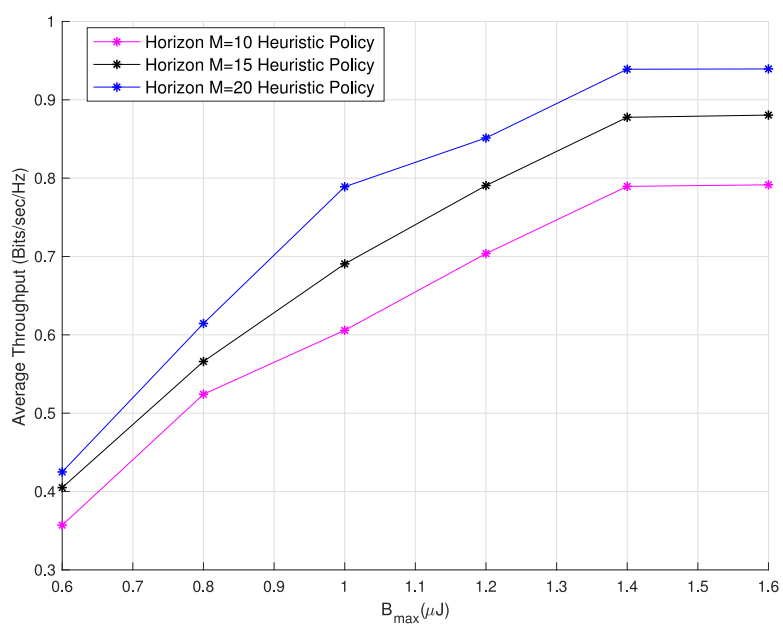

Fig. 9. Average Throughput vs Battery capacity $B_{\max }$ Heuristic Policy for Horizon length $\mathrm{M}=10,15,20$.

less than its counterpart introduced in this paper. This is due to the fact that in our previous work, one additional optimization variable was spectrum sensing decision $\theta_{k}$, which could only

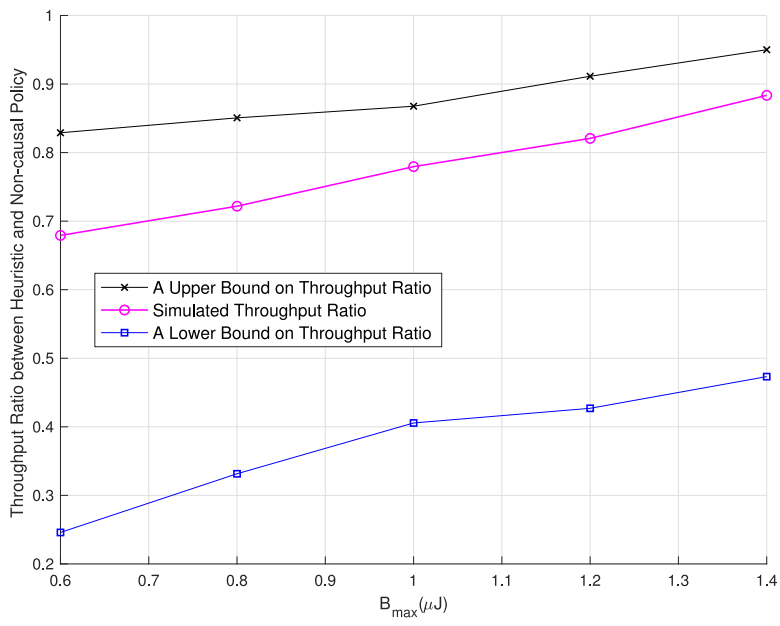

Fig. 10. Throughput Ratio between Heuristic and Non-Causal Policy vs Battery Capacity $B_{\max }$ for Horizon length $\mathrm{M}=4$.

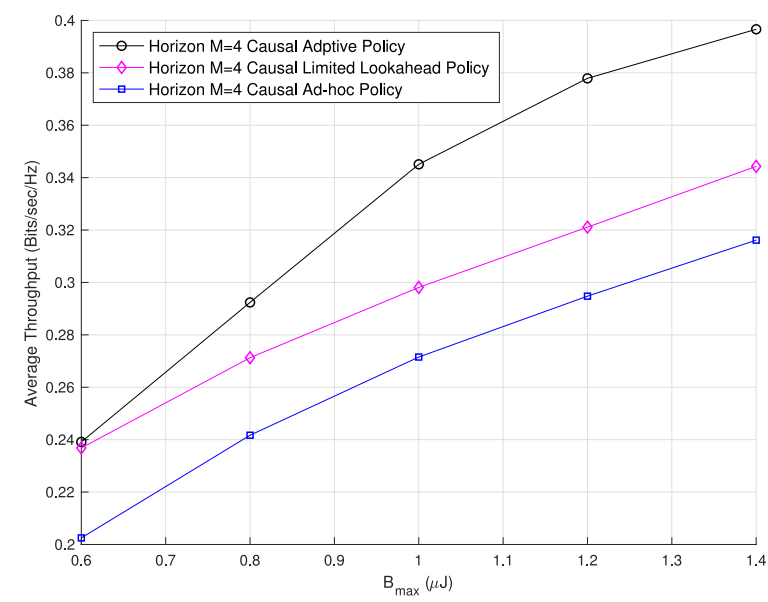

Fig. 11. Average Throughput vs Battery capacity $B_{\max }$ Horizon length $\mathrm{M}=4$ with Causal information for Adaptive, Limited look-ahead and Ad-Hoc Policy.

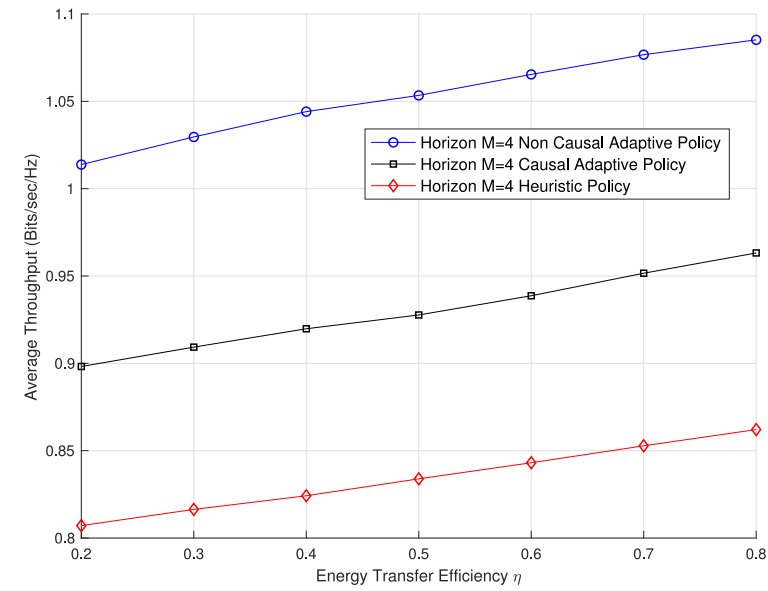

Fig. 12. Average Throughput vs Energy Transfer Efficiency $\eta$ Horizon length M=4 with Non-Causal, Causal and Heuristic Policy.

take binary values, whereas in our present work, the problem formulation includes parameters like probability of detection $P_{d}$ and probability of false alarm $P_{f a}$, which are continuous 
functions of sensing time parameter $\tau_{k}$. We have also simulated comparative plots for Non-causal, causal and heuristic policy when the PU traffic is comparatively light in Fig. 8. For this figure, we have assumed the PU activity probability to be $\mu=0.2$. The plots show that the average throughput for this scenario is higher compared to the scenario involving $\mu=0.8$. This is due to the fact that PU spectrum availability for $\mu=0.2$ is much more significant compared to the case when $\mu=0.8$.

Fig. 10 shows the ratio of average throughput between heuristic and non-causal policy for horizon length $M=4$ with respect to varying level of battery capacity $B_{\max }$. We also provide an upper bound based on a feasible policy for the non-causal case for this ratio of average throughput between the heuristic and the non-causal policy. We assume that the sensing time for every time slot to be $\tau_{k}=\tau_{l}$. We also assume that no energy is shared between the SUs. Assuming that each SU transmits the same amount of power at every time instant, we can derive the following expression as an upper bound of $p_{i, k}$.

$$
\begin{aligned}
p_{i, k} \leq & \min \left\{P_{\text {max }}, \frac{Q_{a v g}}{N \frac{T-\tau_{l}}{T}\left(1-P_{d}\left(\tau_{l}\right)\right) \sum_{k=1}^{M} \sum_{i=1}^{N} g_{i, k}},\right. \\
& \left.\frac{\min \left\{B_{\text {max }}, B_{i}+\sum_{r=1}^{k-1} H_{i, r}-p_{s} \tau_{l}\left(\sum_{r=1}^{k} a_{i, r}\right)\right\}}{k\left(T-\tau_{l}\right)\left(\left(1-P_{d}\left(\tau_{l}\right)\right) \mu+\left(1-P_{f a}\left(\tau_{l}\right)\right)(1-\mu)\right.}\right\}
\end{aligned}
$$

We also assume that at every time slot at least one SU decides to sense, the probability of which is very high when the number of SUs becomes large. This allows us to use the tightest upper bound which satisfies all such $M N$ constraints in (38) for the transmission by all SUs at all time slots. We propose a lower bound on the throughput ratio, by using the upper bound expression for the non-causal policy from equation (27). The expression in (27) assumes that the interference constraint is absent from the optimization problem, resulting in an enlarged feasible set of transmission power and sensing time. Thus the corresponding lower bound is rather loose compared to its upper bound counterpart. The throughput ratio between the Heuristic and Non-causal policy increases gradually with battery capacity $B_{\max }$, because as the battery capacity $B_{\max }$ increases, on average the amount of energy that can be stored without overflow in the battery also increases. This further makes the optimal solutions for non-causal scenario less superior compared to its heuristic counterpart, as both policies have more energy at their disposal for the sensing, transmission and energy sharing process.

In Fig. 11, we compared the average throughput with respect to the battery capacity $B_{\max }$ with causal information and adaptive sensing time, limited look-ahead policy and ad-hoc policy. From the simulation, we notice that for $M=4$ and $B_{\max }=1.4 \mu \mathrm{J}$ the average sum throughput corresponding to causal limited look-ahead policy and ad-hoc policy are 13.7 and 20 percent lower compared to its causal adaptive counterpart respectively. Between the limited look-ahead and ad-hoc policy the first one is more computationally complex, but performs better in terms of average sum throughput, which again illustrates the trade-off between complexity and performance.

In Fig. 12, we use an asymmetric energy harvesting and channel gain model. This situation is different compared to all the previous figures, where we assumed that the mean channel gains $\mu_{g}$ and $\mu_{h}$ and mean harvested energy $\mu_{H}$ are the same for the two SUs. Intuitively, SUs do not have any incentive to share energy with their neighbours if their CSI and ESI are symmetric. We assume that the mean channel gain between the SU transmitters and the PU receiver, $\mu_{g}=1$ for both SUs, but the mean channel gain between the SU transmitters and the FC is different for different SUs. We take $\mu_{h}^{1}=1$ and $\mu_{h}^{2}=10$. The harvested energy mean is taken as $\mu_{H}^{1}=10 \mu \mathrm{J}$ and $\mu_{H}^{2}=1 \mu \mathrm{J}$. The battery capacity is assumed to be $B_{\max }=1.5 \mu J$. The figure shows that the average sum throughput indeed increases with increasing energy transfer efficiency, indicating an increased incentive to share energy in the asymmetric case. This increase is more prominent in the non-causal and causal dynamic programming graphs as compared to the heuristic policy, where the increase is marginal in nature.

\section{CONCLUSION}

This paper deals with a mixed integer non-linear programming problem of maximizing a finite horizon expected achievable sum rate of a cognitive multiple access channel consisting of energy harvesting SUs under long term average interference constraints at the PU with a cooperative spectrum sensing model, where the SUs are also capable of directional energy sharing with their neighbours. We first investigated this optimization problem with non-causal CSI and infinite battery settings, where the integer variable for decision to sense is optimized by an exhaustive search approach when the horizon length and number of SUs are small, and a less computationally complex heuristic policy in the case of longer horizon lengths and moderate to large number of SUs. In the non-causal case, we also provided analytical solutions for the sensing time, transmission power, energy shared. The performance in the non-causal scenario is used to provide an upper bound on the performance of its causal CSI (and ESI) counterpart. The causal CSI and ESI scenario with finite battery setting is handled using dynamic programming techniques. To combat the curse of dimensionality of dynamic programming, we also propose two sub-optimal policies for the scenario involving causal information, namely limited lookahead and ad-hoc policy. Through simulations we found that the energy sharing is only beneficial in the situation involving asymmetric energy harvesting and channel condition scenarios, i.e., when some $\mathrm{SU}$ is harvesting significantly less on average compared to other SUs, but has a better channel for data transmission.

\section{APPENDIX A \\ PROOF OF LEMMA 1}

Since $p_{j, k}^{\star}=0$ and $p_{i, k}^{\star}>0$ from the KKT condition it follows that $\delta_{i, k}^{\star}=0, \alpha_{j, k}^{\star}=0$, and $\delta_{j, k}^{\star} \geq 0, \alpha_{j i k}^{\star} \geq 0$. 
Using the KKT optimality conditions, it can be deduced after some basic algebra that:

$$
\begin{aligned}
& \frac{\left(T-\tau_{k}\right)}{M T \log 2} h_{i, k}\left\{\frac{\mu\left(1-P_{d}\left(\tau_{k}\right)\right)}{\sigma^{2}+\sigma_{i n}^{2}+\sum_{l=1}^{N} p_{l, k}^{\star} h_{l, k}}\right. \\
& \left.+\frac{(1-\mu)\left(1-P_{f a}\left(\tau_{k}\right)\right)}{\sigma^{2}+\sum_{l=1}^{N} p_{l, k}^{\star} h_{l, k}}\right\} \\
& \geq \frac{T-\tau_{k}}{M T} \lambda g_{i, k}\left(1-P_{d}\left(\tau_{k}\right)\right)+\left(T-\tau_{k}\right) \\
& \times\left\{\left(1-P_{d}\left(\tau_{k}\right)\right) \mu+\left(1-P_{f a}\left(\tau_{k}\right)\right)(1-\mu)\right\} \sum_{r=k}^{M} \beta_{i, r}^{\star} \\
& \frac{\left(T-\tau_{k}\right)}{M T \log 2} h_{j, k}\left\{\frac{\mu\left(1-P_{d}\left(\tau_{k}\right)\right)}{\sigma^{2}+\sigma_{i n}^{2}+\sum_{l=1}^{N} p_{l, k}^{\star} h_{l, k}}\right. \\
& \left.+\frac{(1-\mu)\left(1-P_{f a}\left(\tau_{k}\right)\right)}{\sigma^{2}+\sum_{l=1}^{N} p_{l, k}^{\star} h_{l, k}}\right\} \\
& \leq \frac{T-\tau_{k}}{M T} \lambda g_{j, k}\left(1-P_{d}\left(\tau_{k}\right)\right)+\left(T-\tau_{k}\right) \\
& \times\left\{\left(1-P_{d}\left(\tau_{k}\right)\right) \mu+\left(1-P_{f a}\left(\tau_{k}\right)\right)(1-\mu)\right\} \sum_{r=k}^{M} \beta_{j, r}^{\star}
\end{aligned}
$$

The above two equations imply the required result.

\section{APPENDIX B \\ PROOF OF LEMMA 2}

First, we need the following lemma:

Lemma 3: The optimal solution of the problem has at most one user indexed by $i$ that satisfies $0<p_{i, k}^{\star}<P_{\max }$ where $i=\pi(|\mathcal{I}|)$, and the following condition must hold for the optimal transmission power:

$$
\begin{aligned}
\frac{d_{\pi(|\mathcal{I}|), k}}{\lambda e_{\pi(|\mathcal{I}|), k}+f_{\pi(|\mathcal{I}|), k}}=\left\{\frac{\mu\left(1-P_{d}\left(\tau_{k}\right)\right)}{\sigma^{2}+\sigma_{i n}^{2}+\sum_{c=1}^{|\mathcal{I}|} P_{\max } h_{\pi(c), k}}\right. \\
\left.+\frac{(1-\mu)\left(1-P_{f a}\left(\tau_{k}\right)\right)}{\sigma^{2}+\sum_{c=1}^{|\mathcal{I}|} P_{\max } h_{\pi(c), k}}\right\}^{-1} .
\end{aligned}
$$

Proof: (By contradiction) We assume that there exist two users $i$ and $j$ such that with $0<p_{i, k}^{\star}<P_{\max }$ and $0<$ $p_{j, k}^{\star}<P_{\max }$. From the KKT condition we determine that $\delta_{i, k}^{\star}=\delta_{j, k}^{\star}=0$ and $\alpha_{i, k}^{\star}=\alpha_{j, k}^{\star}=0$ respectively. Using these values we can deduce: $\frac{d_{i, k}}{\lambda e_{i, k}+f_{i, k}}=\frac{d_{j, k}}{\lambda e_{j, k}+f_{j, k}}$. Since $h_{i, k}$ and $g_{i, k}$ 's are independent of $h_{j, k}$ and $g_{j, k}$ 's and they are drawn from a continuous distribution and $\lambda$ is constant, it can be inferred that the above equality is satisfied with a probability of measure zero. Thus we can deduce that there is at most one user (say user $i$ ) with $0<p_{i, k}^{\star}<P_{\max }$. Thus the following expression holds for transmission power for $i^{\text {th }} \mathrm{SU}$ :

$$
\begin{aligned}
\frac{d_{i, k}}{\lambda e_{i, k}+f_{i, k}}= & \left\{\frac{\mu\left(1-P_{d}\left(\tau_{k}\right)\right)}{\sigma^{2}+\sigma_{i n}^{2}+\sum_{c=1}^{|\mathcal{I}|} P_{\max } h_{\pi(c), k}}\right. \\
& \left.+\frac{(1-\mu)\left(1-P_{f a}\left(\tau_{k}\right)\right)}{\sigma^{2}+\sum_{c=1}^{|\mathcal{I}|} P_{\max } h_{\pi(c), k}}\right\}^{-1}
\end{aligned}
$$

Using the KKT conditions it is easy to check that for any user $z \in \mathcal{I}, z \neq i$, with $p_{z, k}^{\star}>0$ must satisfy $\frac{d_{z, k}}{\lambda e_{z, k}+f_{z, k}} \geq$ $\frac{d_{i, k}}{\lambda e_{i, k}+f_{i, k}}$. Thus it follows that $i=\pi(|\mathcal{I}|)$.

From this result, we can now infer that there are only two possible sets of solutions for $p_{i, k}^{\star}, k \in \mathcal{I}$ :

- Case I: $p_{\pi(a), k}^{\star}=P_{\max }, a=1,2, \ldots,|\mathcal{I}|$.

- Case II: $p_{\pi(a), k}^{\star}=P_{\max }, a=1,2, \ldots,|\mathcal{I}|-1$

The expression for $p_{\pi(|\mathcal{I}|), k}^{\star}$ can be solved by solving the following non-linear equation:

$$
\left\{\frac{\mu\left(1-P_{d}\left(\tau_{k}\right)\right)}{\rho_{1}+p_{\pi(|\mathcal{I}|), k}^{\star} h_{\pi(|\mathcal{I}|), k}}+\frac{(1-\mu)\left(1-P_{f a}\left(\tau_{k}\right)\right)}{\rho_{2}+p_{\pi(|\mathcal{I}|), k}^{\star} h_{\pi(|\mathcal{I}|), k}}\right\}^{-1}=\omega
$$

where

$$
\begin{aligned}
\rho_{1} & =\sigma^{2}+\sigma_{i n}^{2}+\sum_{c=1}^{|\mathcal{I}|-1} P_{\max } h_{\pi(c), k} \\
\rho_{2} & =\sigma^{2}+\sum_{c=1}^{|\mathcal{I}|-1} P_{\max } h_{\pi(c), k} \\
\omega & =\frac{d_{\pi(|\mathcal{I}|), k}}{\lambda e_{\pi(|\mathcal{I}|), k}+f_{\pi(|\mathcal{I}|), k}}
\end{aligned}
$$

Since $p_{\pi(|\mathcal{I}|), k}^{\star} \leq P_{\text {max }}$, thus we can write the optimal transmission power as $p_{\pi(|\mathcal{I}|), k}^{\star}=\min \left\{P_{\max }, P_{k}^{*}\right\}$, where $P_{k}^{*}$ is the feasible solution to the quadratic equation (43).

All that is required now is to show the fact that optimal number of active users $|\mathcal{I}|$ is the largest value of $x$ such that :

$$
\begin{aligned}
\frac{d_{\pi(x), k}}{\lambda e_{\pi(x), k}+f_{\pi(x), k}}>\left\{\frac{\mu\left(1-P_{d}\left(\tau_{k}\right)\right)}{\sigma^{2}+\sigma_{i n}^{2}+\sum_{c=1}^{x-1} P_{\max } h_{\pi(c), k}}\right. \\
\left.+\frac{(1-\mu)\left(1-P_{f a}\left(\tau_{k}\right)\right)}{\sigma^{2}+\sum_{c=1}^{x-1} P_{\max } h_{\pi(c), k}}\right\}^{-1}
\end{aligned}
$$

It can be shown that both the case I and case II, for any SU $\pi(b), b=1, \ldots,|\mathcal{I}|$, the above inequality holds. Since from Lemma 1, it can be said that its left hand side decreases with $x$, while the right hand side increases with $x$, thus it is sufficient to show that the inequality holds for $b=|\mathcal{I}|$. Thus in this case $\delta_{\pi(|\mathcal{I}|), k}^{\star}=0$ and $\alpha_{\pi(|\mathcal{I}|), k}^{\star} \geq 0$, we have

$$
\begin{aligned}
\frac{d_{\pi(|\mathcal{I}|), k}}{\lambda e_{\pi(|\mathcal{I}|), k}+f_{\pi(|\mathcal{I}|), k}} \geq & \left\{\frac{\mu\left(1-P_{d}\left(\tau_{k}\right)\right)}{\sigma^{2}+\sigma_{i n}^{2}+\sum_{c=1}^{|\mathcal{I}|} p_{|\mathcal{I}|, k}^{\star} h_{\pi(c), k}}\right. \\
& \left.+\frac{(1-\mu)\left(\left(1-P_{f a}\left(\tau_{k}\right)\right)\right)}{\sigma^{2}+\sum_{c=1}^{|\mathcal{I}|} p_{|\mathcal{I}|, k}^{\star} h_{\pi(c), k}}\right\}^{-1}
\end{aligned}
$$




$$
\begin{gathered}
>\left\{\frac{\mu\left(1-P_{d}\left(\tau_{k}\right)\right)}{\sigma^{2}+\sigma_{i n}^{2}+\sum_{c=1}^{|\mathcal{I}|-1} P_{\max } h_{\pi(c), k}}\right. \\
\left.+\frac{(1-\mu)\left(1-P_{f a}\left(\tau_{k}\right)\right)}{\sigma^{2}+\sum_{c=1}^{|\mathcal{I}|-1} P_{\max } h_{\pi(c), k}}\right\}^{-1}
\end{gathered}
$$

[15] S. Lee, R. Zhang, and K. Huang, "Opportunistic wireless energy harvesting in cognitive radio networks," IEEE Trans. Wireless Commun., vol. 12, no. 9, pp. 4788-4799, Sep. 2013.

[16] O. Elnahas, M. Elsabrouty, O. Muta, and H. Furukawa, "Game theoretic approaches for cooperative spectrum sensing in energy-harvesting cognitive radio networks," IEEE Access, vol. 6, pp. 11086-11100, 2018.

[17] H. S. Lee, M. E. Ahmed, and D. I. Kim, "Optimal spectrum sensing policy in RF-powered cognitive radio networks," IEEE Trans. Veh. Technol., vol. 67, no. 10, pp. 9557-9570, Oct. 2018.

Next we have to show that for any user $\pi(j), j=|\mathcal{I}|+$ $1, \ldots, M$, the inequality does not hold. Again it is sufficient to show that it does not hold for $\pi(|\mathcal{I}|+1)$. For that user $\delta_{\pi(|\mathcal{I}|+1), k}^{\star} \geq 0$ and $\alpha_{\pi(|\mathcal{I}|+1), k}^{\star}=0$, we have

$$
\begin{aligned}
\frac{d_{\pi(|\mathcal{I}|+1), k}}{\lambda e_{\pi(|\mathcal{I}|+1), k}+f_{\pi(|\mathcal{I}|+1), k} \leq} & \left\{\frac{\mu\left(1-P_{d}\left(\tau_{k}\right)\right)}{\sigma^{2}+\sigma_{i n}^{2}+\sum_{c=1}^{|\mathcal{I}|} p_{|\mathcal{I}|, k}^{\star} h_{\pi(c), k}}\right. \\
& \left.+\frac{(1-\mu)\left(1-P_{f a}\left(\tau_{k}\right)\right)}{\sigma^{2}+\sum_{c=1}^{|\mathcal{I}|} p_{|\mathcal{I}|, k}^{\star} h_{\pi(c), k}}\right\}^{-1} \\
\leq & \left\{\frac{\mu\left(1-P_{d}\left(\tau_{k}\right)\right)}{\sigma^{2}+\sigma_{i n}^{2}+\sum_{c=1}^{|\mathcal{I}|} P_{\max } h_{\pi(c), k}}\right. \\
& \left.+\frac{(1-\mu)\left(1-P_{f a}\left(\tau_{k}\right)\right)}{\sigma^{2}+\sum_{c=1}^{|\mathcal{I}|} P_{\max } h_{\pi(c), k}}\right\}^{-1}
\end{aligned}
$$

Thus we can determine $|\mathcal{I}|$, and the proof is complete.

\section{REFERENCES}

[1] "Report of the spectrum efficiency working group," FCC Spectr. Policy Task Force, Washington, DC, USA, Rep. 02-135, Nov. 2002.

[2] S. Kim, J. Lee, H. Wang, and D. Hong, "Sensing performance of energy detector with correlated multiple antennas," IEEE Signal Process. Lett., vol. 16, no. 8, pp. 671-674, Aug. 2009.

[3] S. Haykin, "Cognitive radio: Brain-empowered wireless communications," IEEE J. Sel. Areas Commun., vol. 23, no. 2, pp. 201-220, Feb. 2005 .

[4] T. Yucek and H. Arslan, "A survey of spectrum sensing algorithms for cognitive radio applications," IEEE Commun. Surveys Tuts., vol. 11, no. 1, pp. 116-130, 1st Quart., 2009.

[5] K. Cichoń, A. Kliks, and H. Bogucka, "Energy-efficient cooperative spectrum sensing: A survey," IEEE Commun. Surveys Tuts., vol. 18, no. 3, pp. 1861-1886, 3rd Quart., 2006.

[6] C. Han et al., "Green radio: Radio techniques to enable energy-efficient wireless networks," IEEE Commun. Mag., vol. 49, no. 6, pp. 46-54, Jun. 2011.

[7] L. X. Cai et al., "Dimensioning network deployment and resource management in green mesh networks," IEEE Wireless Commun., vol. 18, no. 5, pp. 58-65, Oct. 2011.

[8] S. Ulukus et al., "Energy harvesting wireless communications: A review of recent advances," IEEE J. Sel. Areas Commun., vol. 33, no. 3, pp. 360-381, Mar. 2015.

[9] J. Yang and S. Ulukus, "Optimal packet scheduling in an energy harvesting communication system," IEEE Trans. Commun., vol. 60, no. 1, pp. 220-230, Jan. 2012.

[10] K. Tutuncuoglu and A. Yener, "Optimum transmission policies for battery limited energy harvesting nodes," IEEE Trans. Wireless Commun., vol. 11, no. 3, pp. 1180-1189, Mar. 2012.

[11] H. Li, N. Jaggi, and B. Sikdar, "Relay scheduling for cooperative communications in sensor networks with energy harvesting," IEEE Trans. Wireless Commun., vol. 10, no. 9, pp. 2918-2928, Sep. 2011.

[12] M. A. Antepli, E. Uysal-Biyikoglu, and H. Erkal, "Optimal packet scheduling on an energy harvesting broadcast link," IEEE J. Sel. Areas Commun., vol. 29, no. 8, pp. 1721-1731, Sep. 2011.

[13] H. Huang and V. K. N. Lau, "Decentralized delay optimal control for interference networks with limited renewable energy storage," IEEE Trans. Signal Process., vol. 60, no. 5, pp. 2552-2561, May 2012.

[14] J. Yang and S. Ulukus, "Optimal packet scheduling in a multiple access channel with rechargeable nodes," in Proc. IEEE Int. Conf. Commun. (ICC), Jun. 2011, pp. 1-5.
[18] J. Ren et al., "RF energy harvesting and transfer in cognitive radio sensor networks: Opportunities and challenges," IEEE Commun. Mag., vol. 56, no. 1, pp. 104-110, Jan. 2018.

[19] R. Zhang, S. Cui, and Y.-C. Liang, "On ergodic sum capacity of fading cognitive multiple-access and broadcast channels," IEEE Trans. Inf. Theory, vol. 55, no. 11, pp. 5161-5178, Nov. 2009.

[20] D. Xu and Q. Li, "Joint power control and time allocation for wireless powered underlay cognitive radio networks," IEEE Wireless Commun. Lett., vol. 6, no. 3, pp. 294-297, Jun. 2017.

[21] Y.-C. Liang, Y. Zeng, E. C. Y. Peh, and A. T. Hoang, "Sensingthroughput tradeoff for cognitive radio networks," IEEE Trans. Wireless Commun., vol. 7, no. 4, pp. 1326-1337, Apr. 2008.

[22] O. Ozel and S. Ulukus, "Information-theoretic analysis of an energy harvesting communication system," in Proc. IEEE 21st Int. Symp. Pers. Indoor Mobile Radio Commun. Workshops, Sep. 2010, pp. 330-335.

[23] S. Park and D. Hong, "Achievable throughput of energy harvesting cognitive radio networks," IEEE Trans. Wireless Commun., vol. 13, no. 2, pp. 1010-1022, Feb. 2014

[24] S. Park, H. Kim, and D. Hong, "Cognitive radio networks with energy harvesting," IEEE Trans. Wireless Commun., vol. 12, no. 3, pp. 1386-1397, Mar. 2013.

[25] S. Knorn, S. Dey, A. Ahlén, and D. E. Quevedo, "Distortion minimization in multi-sensor estimation using energy harvesting and energy sharing," IEEE Trans. Signal Process., vol. 63, no. 11, pp. 2848-2863, Jun. 2015.

[26] Y. H. Bae and J. W. Baek, "Sensing strategy exploiting channel memory in CR network with RF energy harvesting," IEEE Commun. Lett., vol. 22, no. 12, pp. 2539-2542, Dec. 2018.

[27] S. Yin, Z. Qu, and S. Li, "Achievable throughput optimization in energy harvesting cognitive radio systems," IEEE J. Sel. Areas Commun., vol. 33, no. 3, pp. 407-422, Mar. 2015.

[28] Z. Li, B. Liu, J. Si, and F. Zhou, "Optimal spectrum sensing interval in energy-harvesting cognitive radio networks," IEEE Trans. Cogn. Commun. Netw., vol. 3, no. 2, pp. 190-200, Jun. 2017.

[29] A. Celik, A. Alsharoa, and A. E. Kamal, "Hybrid energy harvestingbased cooperative spectrum sensing and access in heterogeneous cognitive radio networks," IEEE Trans. Cogn. Commun. Netw., vol. 3, no. 1, pp. 37-48, Mar. 2017.

[30] A. Celik and A. E. Kamal, "Multi-objective clustering optimization for multi-channel cooperative spectrum sensing in heterogeneous green CRNs," IEEE Trans. Cogn. Commun. Netw., vol. 2, no. 2, pp. 150-161, Jun. 2016.

[31] A. Celik and A. E. Kamal, "Green cooperative spectrum sensing and scheduling in heterogeneous cognitive radio networks," IEEE Trans. Cogn. Commun. Netw., vol. 2, no. 3, pp. 238-248, Sep. 2016.

[32] T. Zhang, W. Chen, and F. Yang, "Balancing delay and energy efficiency in energy harvesting cognitive radio networks: A stochastic Stackelberg game approach," IEEE Trans. Cogn. Commun. Netw., vol. 3, no. 2, pp. 201-216, Jun. 2017.

[33] G. Han, J.-K. Zhang, and X. Mu, "Joint optimization of energy harvesting and detection threshold for energy harvesting cognitive radio networks," IEEE Access, vol. 4, pp. 7212-7222, 2016.

[34] F. Mohammadian, Z. Pourgharehkhan, A. Taherpour, and T. Khattab, "Optimal collaborative energy harvesting spectrum sensing with limited time resource," in Proc. IEEE Wireless Commun. Netw. Conf., Apr. 2016, pp. $1-7$.

[35] X. Liu, W. Lu, L. Ye, F. Li, and D. Zou, "Joint resource allocation of spectrum sensing and energy harvesting in an energy-harvestingbased cognitive sensor network," Sensors, vol. 17, no. 3, p. E600, 2017. [Online]. Available: http://www.mdpi.com/1424-8220/17/3/600

[36] X. Liu, W. Lu, F. Li, M. Jia, and X. Gu, "Optimal simultaneous multislot spectrum sensing and energy harvesting in cognitive radio," in Proc. IEEE Glob. Commun. Conf. (GLOBECOM), Dec. 2016, pp. 1-6.

[37] Z. Wang, V. Aggarwal, and X. Wang, "Iterative dynamic water-filling for fading multiple-access channels with energy harvesting," IEEE $J$. Sel. Areas Commun., vol. 33, no. 3, pp. 382-395, Mar. 2015. 
[38] Z. Wang, V. Aggarwal, and X. Wang, "Power allocation for energy harvesting transmitter with causal information," IEEE Trans. Commun., vol. 62, no. 11, pp. 4080-4093, Nov. 2014.

[39] S. Biswas, A. Shirazinia, and S. Dey, "Sensing throughput optimization in cognitive fading multiple access channels with energy harvesting secondary transmitters," in Proc. 24th Eur. Signal Process. Conf. (EUSIPCO), Aug. 2016, pp. 577-581.

[40] X. Chen, C. Yuen, and Z. Zhang, "Wireless energy and information transfer tradeoff for limited-feedback multiantenna systems with energy beamforming," IEEE Trans. Veh. Technol., vol. 63, no. 1, pp. 407-412, Jan. 2014.

[41] L. Liu, R. Zhang, and K.-C. Chua, "Multi-antenna wireless powered communication with energy beamforming," IEEE Trans. Commun., vol. 62, no. 12, pp. 4349-4361, Dec. 2014.

[42] C. Floudas, Nonlinear and Mixed-Integer Optimization. Fundamentals and Applications. Oxford, U.K.: Oxford Univ. Press, 1995.

[43] K. G. Murty and S. N. Kabadi, "Some NP-complete problems in quadratic and nonlinear programming," Math. Program., vol. 39, no. 2, pp. 117-129, Jun. 1987.

[44] S. Atapattu, C. Tellambura, and H. Jiang, "Energy detection based cooperative spectrum sensing in cognitive radio networks," IEEE Trans. Wireless Commun., vol. 10, no. 4, pp. 1232-1241, Apr. 2011.

[45] X. Kang, Y.-C. Liang, A. Nallanathan, H. K. Garg, and R. Zhang, "Optimal power allocation for fading channels in cognitive radio networks: Ergodic capacity and outage capacity," IEEE Trans. Wireless Commun., vol. 8, no. 2, pp. 940-950, Feb. 2009.

[46] T. Cover, R. McEliece, and E. Posner, "Asynchronous multipleaccess channel capacity," IEEE Trans. Inf. Theory, vol. IT-27, no. 4, pp. 409-413, Jul. 1981.

[47] J. Lee and S. Leyffer, Eds., Mixed Integer Nonlinear Programming. New York, NY, USA: Springer, 2012.

[48] S. Boyd and L. Vandenberghe, Convex Optimization. Cambridge, U.K.: Cambridge Univ. Press, 2004.

[49] D. P. Bertsekas, Dynamic Programming and Optimal Control, vol. 1 , 3rd ed. Belmont, MA, USA: Athena Sci., 2005.

[50] A. Goldsmith, Wireless Communications. New York, NY, USA: Cambridge Univ. Press, 2005.

[51] M. Schlueter, S. O. Erb, M. Gerdts, S. Kemble, and J.-J. Rückmann, "MIDACO on MINLP space applications," Adv. Space Res., vol. 51, no. 7, pp. 1116-1131, 2013.

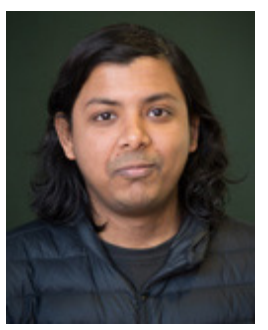

Sinchan Biswas was born in Halisahar, India, in 1988. He received the M.Tech. degree from the Department of Electrical Engineering, Indian Institute of Technology Kanpur in 2013. He is currently pursuing the Ph.D. degree with the Department of Engineering Sciences, Uppsala University, Sweden. From 2013 to 2014, he was a Research Assistant with the Indian Institute of Technology Kanpur. His research interests include signal processing, information theory, and stochastic control.

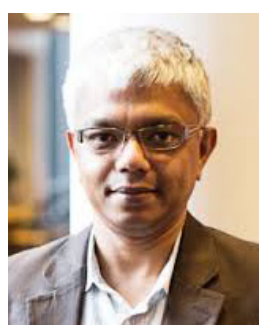

Subhrakanti Dey received the Bachelor of Technology and Master of Technology degrees from the Department of Electronics and Electrical Communication Engineering, Indian Institute of Technology Kharagpur, Kharagpur, in 1991 and 1993, respectively, and the Ph.D. degree from the Department of Systems Engineering, Research School of Information Sciences and Engineering, Australian National University, Canberra, in 1996.

He was a Professor with the Department of Electrical and Electronic Engineering, University of Melbourne, Parkville, Australia, from 2000 to 2013, and a Professor of telecommunications with the University of South Australia from 2017 to 2018. $\mathrm{He}$ is currently a Professor with the Hamilton Institute, National University of Ireland, Maynooth, Ireland, and with the Department of Engineering Sciences, Uppsala University, Sweden. From 1995 to 1997 and 1998 to 2000, he was a Post-Doctoral Research Fellow with the Department of Systems Engineering, Australian National University. From 1997 to 1998, he was a Post-Doctoral Research Associate with the Institute for Systems Research, University of Maryland, College Park. His current research interests include wireless communications and networks, signal processing for sensor networks, networked control systems, and molecular communication systems.

Prof. Dey currently serves on the Editorial Board for the IEEE Control Systems Letters, the IEEE Transactions on Control of Network Systems, and the IEEE TRANSACTIONS ON WirElESS COMMUNiCATIONS. He was also an Associate Editor for the IEEE TRANSACTIONS ON Signal PROCESSING from 2007 to 2010 and 2014 to 2018, the IEEE Transactions on Automatic Control from 2004 to 2007, and Systems and Control Letters (Elsevier) from 2003 to 2013.

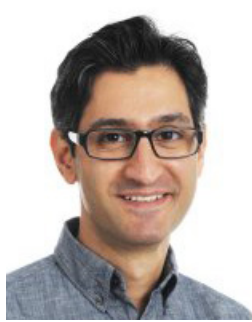

Amirpasha Shirazinia received the Ph.D. degree in telecommunications from the KTH Royal Institute of Technology, Stockholm, Sweden, in 2014. He was a Post-Doctoral Research Fellow with Uppsala University from 2014 to 2016. He then joined Ericsson Research, Stockholm, in 2016. He has extensive experience in research, simulation, and standardization within 4G (LTE)/5G (NR). He joined AI and Analytics Team with Swedbank in 2018 as a Senior Data Scientist. His interests span machine learning, optimization, recommender systems, data analysis, statistical data and signal processing, optimization, coding, and wireless communications. 